\title{
Herakles - Herakleios - Christus. Georgios Pisides und der kosmorhýstes
}

In den Jahren um 630 - nach dem byzantinischen Sieg über die Sāsāniden, aber noch vor dem Beginn der Auseinandersetzungen mit den muslimischen Arabern - verfasste Theophylakt Simokates sein Geschichtswerk über die Herrschaft des Kaisers Maurikios (582-602). ${ }^{1}$ Der eigentlichen Darstellung vorangestellt ist ein rhetorisch überhöhter, pathetischer Dialog zwischen den Personifikationen der Philosophie und der Geschichte, in dem beide ihre Wiederauferstehung feiern, nachdem sie unter dem „Ty-

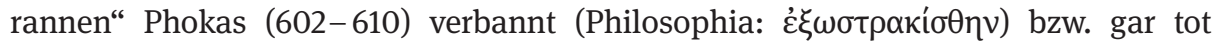

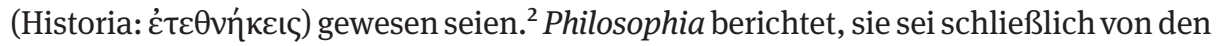
„Herakliden“ gerettet worden, und spielt damit recht eindeutig auf den Sturz und die Ermordung des Phokas durch Herakleios (610 -641) und seine Anhänger an. ${ }^{3}$ Doch auch Historia weiß ihren Wohltäter zu benennen: „den großen Erzpriester und Vor-

\footnotetext{
$1 \mathrm{Zu}$ Theophylakt und seinem Geschichtswerk ist grundlegend die Monographie von Mi. Whitby, The Emperor Maurice and His Historian: Theophylact Simocatta on Persian and Balkan Warfare, Oxford 1988; s. auch D. Brodka, Die Geschichtsphilosophie in der spätantiken Historiographie. Studien zu Prokopios von Kaisareia, Agathias von Myrina und Theophylaktos Simokattes, Frankfurt a.M./Berlin u. a. 2004; wichtig sind überdies O. Veh, Untersuchungen zu dem byzantinischen Historiker Theophylaktos Simokattes. Wissenschaftliche Beilage zum Jahresbericht 1956/57 des Human. Gymnasiums Fürth i. Bay., Fürth 1957; Mi. Whitby, Greek Historical Writing after Procopius: Variety and Vitality, in: Av. Cameron/L. I. Conrad (Hgg.), The Byzantine and Early Islamic Near East I: Problems in the Literary Source Material, Princeton (N.J.) 1992, 25-80, bes. 45-54; Mi. Whitby/Ma. Whitby, The History of Theophylact Simocatta. An English Translation with Introduction and Notes, Oxford 1986, ND 1988; P. Schreiner, Theophylakt Simokates. Geschichte. Übersetzt und erläutert, Stuttgart 1985; J. D. C. Frendo, History and Panegyric in the Age of Heraclius: The Literary Background to the Composition of the Histories of Theophylact Simocatta, DOP 42 (1988), 143-156; S. Efthymiadis, A Historian and His Tragic Hero: A Literary Reading of Theophylact Simokatta's Ecumenical History, in: R. Macrides (Hg.), History as Literature in Byzantium, Farnham/Burlington 2010, 169-185. Einige einleitende Bemerkungen auch bei M. Meier, Prokop, Agathias, die Pest und das ,Ende‘ der antiken Historiographie. Naturkatastrophen und Geschichtsschreibung in der ausgehenden Spätantike, HZ 278 (2004), 281310, hier 304-310. - Zur Datierung des Geschichtswerks s. etwa Schreiner, a.a.O., 2f.; 13; Whitby, Emperor, 39f.; Whitby/Whitby, a.a.O., XVI. Die düstere Grundstimmung des Werks und vermeintliche Anspielungen auf die arabischen Angriffe seit Mitte der 630er Jahre haben neuerdings Efthymiadis, a. a.O., 171; 180, zu Spekulationen über eine mögliche Entstehungszeit in der 2. Hälfte der 630er Jahre veranlasst. Die von ihm herangezogenen Anspielungen sind indes alles andere als klar, und für die ,Grundstimmung' des Werkes (die zunächst einmal nur aus der Perspektive moderner Einschätzungen als düster erscheint) lassen sich auch andere Erklärungen beibringen, z. B. die mögliche Intention, das Schicksal des Maurikios möglichst tragisch und ergreifend als an den aktuellen Kaiser gerichtete Mahnung zu zeichnen.
}

2 Theophyl. Sim. Dial. 4-5.

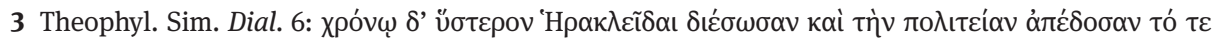

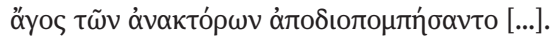


steher der gesamten Oikoumene“. ${ }^{4}$ Man hat damit verschiedentlich Sergios, den Patriarchen von Konstantinopel (610-638), identifizieren wollen. ${ }^{5}$ Das aber ist wenig wahrscheinlich, denn im Folgenden vergleicht die Figur der Historia ihre Wiedererweckung explizit mit der Rückführung der Alkestis aus dem Totenreich durch Herakles $^{6}$ und verwendet damit ein Bild, das sich (ebenfalls unter Hinweis auf die Oikoumene) auch beim zeitgenössischen Dichter Georgios Pisides findet - und zwar mit ausdrücklichem Bezug auf Herakleios. ${ }^{7}$ Dass hinter dem „großen Erzpriester und Vorsteher der gesamten Oikoumene“ denn auch tatsächlich der Kaiser, und nicht der Patriarch, zu sehen ist, wird bereits aus dem Umstand ersichtlich, dass die im Dialog unmittelbar vorausgehende Erwähnung der Herakliden (s.o.) die Parallele HeraklesHerakleios für den weiteren Gesprächsverlauf geradezu festschreibt. ${ }^{8}$

Mit dieser an prominenter Stelle vorgetragenen Analogie zwischen dem aktuellen Kaiser und dem mythischen Helden steht Theophylakt nicht allein. Namentlich sein Zeitgenosse Georgios Pisides hat wiederholt das Herakles-Exempel in Verbindung mit Herakleios evoziert, in unterschiedlichen Kontexten, zumeist explizit, mitunter aber auch vermittelt durch subtile Anspielungen - ja, selbst eine Passage beim sog. Fredegar könnte auf eventuelle Herakles-Herakleios-Bezüge hindeuten. ${ }^{9}$ Man kann es sich leicht machen und ganz simple Gründe für dieses Phänomen anbringen: Zunächst natürlich den Namen des Kaisers, der die Herakles-Assoziation geradezu vorgegeben haben muss; dann die generelle Vorliebe des Georgios für mythologische Beispiele und Analogien, ${ }^{10}$ weiterhin die traditionelle Prominenz des Herakles-Themas in der (spät) antiken Panegyrik ${ }^{11}$ und herrscherlichen Ikonographie, ${ }^{12}$ ferner die Herakles-Asso-

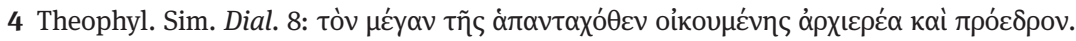

5 So etwa Schreiner (s. Anm. 1), 240 f., Anm. 12; Whitby, Emperor (s. Anm. 1), 40; 44; Whitby/Whitby (s. Anm. 1), 4, Anm. 9.

6 Theophyl. Sim. Dial. 9.

7 Georg. Pisid. Her. 1,71-73.

8 Für diese Identifikation des „großen Erzpriesters“ mit Kaiser Herakleios plädiert neben H. Hunger, Die hochsprachliche profane Literatur der Byzantiner, Bd. 1-2, München 1978, I 315, mit großem Nachdruck auch Frendo (s. Anm. 1), 144f., Anm. 11, der zudem darauf hinweist, dass in der Spätantike

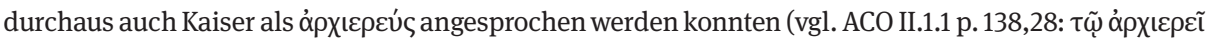

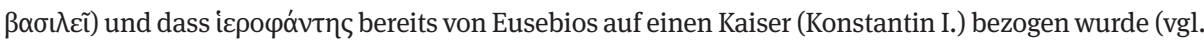
Euseb. Triak. 3; VC 4,22). Trotz der klaren Argumente Frendos versucht allerdings Whitby, Greek Historical Writing (s. Anm. 1), 47, mit Anm. 97, die Sergios-Identifikation weiterhin aufrecht zu erhalten. 9 Fredeg. 65; dazu S. Esders, Herakleios, Dagobert und die „beschnittenen Völker“. Die Umwälzungen des Mittelmeerraums im 7. Jahrhundert in der Chronik des sog. Fredegar, in: A. Goltz/H. Leppin/ H. Schlange-Schöningen (Hgg.), Jenseits der Grenzen. Beiträge zur spätantiken und frühmittelalterlichen Geschichtsschreibung, Berlin/New York 2009, 239-311, hier 290.

10 Dazu s. etwa Ma. Whitby, A New Image for a New Age: George of Pisidia on the Emperor Heraclius, in: E. Dabrowa (Hg.), The Roman and Byzantine Army in the East, Krakau 1994, 197- 225.

11 Vgl. dazu Th. Nissen, Historisches Epos und Panegyrikos in der Spätantike, Hermes 75 (1940), 298 325, hier 302-307. Menander Rhetor (D. A. Russell/N. G. Wilson, Menander Rhetor. Edited with Translation and Commentary, Oxford 1981, p. 80) hat den Herakles-Vergleich sogar als probates Mittel, 
ziationen früherer Herrscher (insbesondere Commodus sowie die Angehörigen der Tetrarchie) ${ }^{13}$ und schließlich eine mögliche ,Herakles-Mode' in gebildeten Kreisen der Zeit - letzteres jedenfalls könnte ein zu Beginn des 20. Jahrhunderts entdecktes anonymes Gedicht über die Taten des Herakles nahelegen, das vor allem aus metrischen Gründen in das 7. Jahrhundert datiert wird, ${ }^{14}$ oder auch ein Papyrusfund mit einem Schultext, der (wahrscheinlich) die an den Schüler gerichtete Aufforderung enthält, Herakles mit dem Kaiser zu vergleichen. ${ }^{15}$ All dies mag eine Rolle gespielt haben.

Im Folgenden möchte ich jedoch versuchen aufzuzeigen, dass dem HeraklesThema im speziellen Fall des Herakleios wohl noch eine weitere, tiefgründigere Funktion zukommt: Offenkundig war dieser Kaiser nämlich darum bemüht, in der Repräsentation mit Christus assoziiert zu werden (ich formuliere diesen Sachverhalt bewusst ein wenig sperrig, weil die Evidenz schmal ist und nicht immer zwischen Ursache und Wirkung, zwischen gezielten Maßnahmen und situativen Reaktionen, differenziert werden kann). Eine plumpe, explizit artikulierte Parallelisierung oder gar Gleichsetzung des Kaisers mit Christus hätte den Zeitgenossen des 7. Jahrhunderts indes wohl allzu viel abverlangt - wenngleich die Augusti seit Konstantin wiederholt auf diesem Feld experimentiert haben. ${ }^{16}$ Die Bezüge ließen sich nur behutsam, vornehmlich in spezifischen Brechungen und häufig indirekt vermitteln und durften nicht erkennbar vom Kaiser selbst forciert werden. An diesem Punkt gewinnt die Panegyrik an Bedeutung, da sie Herrscherbilder transportiert, diese aber zugleich auch stets mit besonderen Erwartungen, die an den Kaiser herangetragen werden, auszubalancieren

ein Herrscherlob zu beginnen, empfohlen. Georgios Pisides ist ihm in seinem Panegyricus In Bonum Patricium darin gefolgt.

12 Vgl. A. Grabar, L'empereur dans l'art byzantin, Strasburg 1936, ND London 1971, 93f.; $141 \mathrm{f}$.

13 Commodus: O. Hekster, Commodus. An Emperor at the Crossroads, Amsterdam 2002; E. MeyerZwiffelhoffer, Ein Visionär auf dem Thron? Kaiser Commodus, Hercules Romanus, Klio 88 (2006), 189215. Tetrarchie: F. Kolb, Diocletian und die erste Tetrarchie. Improvisation oder Experiment in der Organisation monarchischer Herrschaft?, Berlin/New York 1987. Generell zur Funktion des Herakles als Bezugsfigur für antike Herrscher: U. Huttner, Die politische Rolle der Heraklesgestalt im griechischen Herrschertum, Stuttgart 1997.

14 B. Knös, Ein spätgriechisches Gedicht über die Arbeiten des Herakles, BZ 17 (1908), 397-429 (Edition); M. L. West, Greek Metre, Oxford 1988, 184, Anm. 72; Whitby (s. Anm. 10), 206.

15 H. Harrauer/P. J. Sijpesteijn, Neue Texte aus dem antiken Unterricht, Wien 1985, 125-136, Nr. 134, Taf. 60; B. Palme, Aufsatzthemen aus dem Schulunterricht (P. Rain. Unterricht 134), in: M. Puhle/ G. Köster (Hgg.), Otto der Große und das Römische Reich. Kaisertum von der Antike zum Mittelalter. Ausstellungskatalog. Landesausstellung Sachsen-Anhalt aus Anlass des 1100. Geburtstages Ottos des Großen, Regensburg/Magdeburg 2012, 338.

16 Vgl. etwa zu Konstantin S. Rebenich, Vom dreizehnten Gott zum dreizehnten Apostel? Der tote Kaiser in der Spätantike, ZAC 4 (2000), 300 - 324, ND in: H. Schlange-Schöningen (Hg.), Konstantin und das Christentum, Darmstadt 2007, 216-244; zu Justinian s. M. Meier, Das andere Zeitalter Justinians. Kontingenzerfahrung und Kontingenzbewältigung im 6. Jahrhundert n.Chr., Göttingen ${ }^{2} 2004$, 547 ff.; allgemein etwa M. Büchsel, Die Entstehung des Christusporträts. Bildarchäologie statt Bildhypnose, Mainz 22004; M. Meier, Göttlicher Kaiser und christlicher Herrscher? Die christlichen Kaiser der Spätantike und ihre Stellung zu Gott, Das Altertum 48 (2003), 129-160. 
hat und somit eine zentrale, jedoch hochkomplexe Quellengattung konstituiert, die kommunikative Prozesse zwischen Herrschern und Beherrschten spiegelt. Georgios Pisides, dessen panegyrische Dichtungen ohnehin zu den wichtigsten Dokumenten aus der Zeit des Herakleios zählen, drängt sich vor diesem Hintergrund als Untersuchungsobjekt geradezu auf. An seinem Beispiel möchte ich aufzeigen, wie das auf den ersten Blick ausgesprochen traditionelle und im Kontext anderer mythischer Bezugsgrößen nicht eben originell erscheinende Herakles-Exempel mitunter in spezifischer Weise aufgeladen werden und dadurch zum indirekten Vermittler einer KaiserChristus-Parallele avancieren konnte. $\mathrm{Zu}$ diesem Zweck werde ich in fünf Schritten vorgehen: (1.) Nach einigen einleitenden Bemerkungen zu Georgios Pisides und seinem Werk werde ich (2.) zentrale Passagen, in denen der Dichter das Herakles-Thema anstimmt, vorstellen. Auf der Grundlage dieses Materials ist danach (3.) grundsätzlich über die Herakleios-Christus-Parallelen und (4.) die Möglichkeiten, die das HeraklesExempel dabei bot, zu reflektieren, bevor schließlich (5.) kurz die Frage zu diskutieren sein wird, warum gerade Herakleios in besonderer Intensität die Christus-Assoziation betreiben ließ.

\section{1.}

Das moderne Urteil über Georgios Pisides ist gespalten. ${ }^{17}$ Galt er der älteren Forschung mitunter noch als Epigone, an dem sich der vermeintliche Niedergang der spätantiken Dichtung festmachen ließ, ${ }^{18}$ so wird neuerdings seine besondere Mittlerstellung

17 Die Forschung zu Georgios Pisides hat vor allem den Arbeiten Mary Whitbys entscheidende Impulse zu verdanken, vgl. Whitby (s. Anm. 10); dies., The Devil in Disguise: The End of George of Pisidia's Hexaemeron Reconsidered, JHS 105 (1995), 115-129; dies., Defender of the Cross: George of Pisidia on the Emperor Heraclius and His Deputies, in: dies. (Hg.), The Propaganda of Power. The Role of Panegyric in Late Antiquity, Leiden/Boston/Köln 1998, 247-273; dies., George of Pisidia's Presentation of the Emperor Heraclius and His Campaigns. Variety and Development, in: G. J. Reinink/B. H. Stolte (Hgg.), The Reign of Heraclius (610 - 641). Crisis and Confrontation, Leuven/Paris/Dudley (Ma.) 2002, 157-173; dies., George of Pisidia and the Persuasive Word:Words, Words, Words ..., in: E. Jeffreys (Hg.), Rhetoric in Byzantium, Aldershot/Burlington 2003, 173-186; s. ferner Nissen (s. Anm. 11), 301-325; J. D. C. Frendo, The Poetic Achievement of George of Pisidia, in: A. Moffatt (Hg.), Maistor. Classical, Byzantine and Renaissance Studies for Robert Browning, Canberra 1984, 159-187; C. Ludwig, Kaiser Herakleios, Georgios Pisides und die Perserkriege, in: Varia III (= Poikila Byzantina 11), Bonn 1991, 73-128; D. M. Olster, Roman Defeat, Christian Response, and the Literary Construction of the Jew, Philadelphia 1994, bes. 51-71 (vgl. bes. ebd., 64f., Anm. 1, mit Angaben zur älteren Literatur); ders., The Date of George of Pisidia's Hexaemeron, DOP 45 (1991), 159-172, hier 159, Anm. 1 (Literatur); F. R. Gahbauer, Georg der Pisidier, in: S. Döpp/W. Geerlings (Hgg.), Lexikon der antiken christlichen Literatur, Freiburg/Basel/ Wien ${ }^{3} 2002$, 285; zuletzt J. Howard-Johnston, Witnesses to a World Crisis. Historians and Histories of the Middle East in the Seventh Century, Oxford 2010, 16 -35; aktuelle Edition mit Einführung, Kommentar und italienischen Übersetzungen: L. Tartaglia (Ed.), Carmi di Giorgio di Pisidia, Turin 1998.

18 Vgl. etwa die unterkühlte Beurteilung des Georgios bei H.-G. Beck, Kirche und theologische Literatur im byzantinischen Reich, München 1959, 448f. - Hunger (s. Anm. 8), II 112f., erkennt zwar die „literaturhistorische [...] Bedeutung des Pisides“ an, hebt ansonsten aber insbesondere die „propa- 
zwischen antiker und mittelalterlicher Literatur betont ${ }^{19}$ und auf seine außerordentlichen poetischen Qualitäten hingewiesen. ${ }^{20}$ Seine Werke gelten mittlerweile nicht nur als zentrale Dokumente für die Herrschaftszeit des Herakleios, sondern auch als Zeugnisse höchster literarischer Kunstfertigkeit. ${ }^{21}$ Zuletzt konnte James HowardJohnston Georgios Pisides gar als einen ,poet of rare talent, with relatively few peers in classical antiquity“ bezeichnen. ${ }^{22}$

Georgios verfasste Werke panegyrisch-historischen und theologischen Inhalts, und in diese Kategorien wurden seine Dichtungen bis vor wenigen Jahren auch stets geschieden - wobei man davon ausging, dass zunächst (bis etwa 630) seine panegyrischen Schöpfungen, danach die theologischen entstanden seien. ${ }^{23}$ Mittlerweile betont die Forschung hingegen recht einmütig, dass sich Theologie und Panegyrik (und damit die Reflexion aktueller politischer Inhalte) bei Georgios kaum trennen lassen. ${ }^{24}$ Und in der Tat greift beides - wie bei einem oströmischen Dichter des frühen 7. Jahrhunderts ja auch gar nicht anders zu erwarten - bei ihm stets ineinander. Ob man Georgios tendenziell eher als Panegyriker (so etwa Theodor Nissen) oder als Theologen sehen möchte (so etwa Mary Whitby oder Irene Huber), ist daher letztlich eine akademische Frage aus moderner Perspektive. ${ }^{25}$

gandistische Tendenz seiner Epik“ als Charakteristikum hervor. Der „reiche mythologische Apparat“ diene vor allem „der Verherrlichung des gottgleichen Kaisers“. Weitestgehend positive Einschätzungen allerdings schon bei K. Krumbacher, Geschichte der byzantinischen Litteratur von Justinian bis zum Ende des Oströmischen Reiches (527-1453). Zweite Auflage bearbeitet unter Mitwirkung von A. Erhard/ H. Gelzer, München 1897, 709-712, vgl. bes. 709 (,der beste Profandichter der byzantinischen Zeit“); O. Bardenhewer, Geschichte der altkirchlichen Literatur, Bd. 5: Die letzte Periode der altkirchlichen Literatur mit Einschluß des ältesten armenischen Schrifttums, Darmstadt ${ }^{2} 2007$ (ursprünglich Freiburg 1932), $168-173$.

19 Etwa von Hunger (s. Anm. 8), II 167 („Mann des Übergangs“); Frendo (s. Anm. 17), 162; ders., Classical and Christian Influences in the Heracliad of George of Pisidia, The Classical Bulletin 62.4 (1986), 53-62, bes. 53; 61; P. Schreiner, G. Pisides, in: LexMa 4, München/Zürich 1989, 1287 f., hier 1288; Olster, Roman Defeat (s. Anm. 17), 51-71; Whitby, Presentation (s. Anm. 17), 173; dies., Persuasive Word (s. Anm. 17), 174; J. O. Rosenqvist, Die byzantinische Literatur. Vom 6. Jahrhundert bis zum Fall Konstantinopels 1453, Berlin/New York 2007, 23.

20 Vgl. bes. Howard-Johnston (s. Anm. 17), 26-31.

21 Beide Aspekte hebt Howard-Johnston (s. Anm. 17), 16-35, hervor.

22 Howard-Johnston (s. Anm. 17), 27; ähnlich ebd., 16.

23 Dieses Verständnis liegt z.B. noch der (unvollendet gebliebenen) Georgios-Edition Pertusis zugrunde, der sein Augenmerk zunächst auf die ,panegyrischen' Gedichte richtete (und zur Herausgabe der ,theologischen` nicht mehr kam), vgl. G. Pertusi, Giorgio di Pisidia. Poemi, I: Panegirici epici. Edizione critica, traduzione e commento, Ettal 1959, mit der Rez. von I. Dujčev, BZ 57 (1964), 412- 416. 24 S. etwa Frendo (s. Anm. 17), 179 ff.; Olster, Date (s. Anm. 17), 159; Whitby (s. Anm. 10), 201; dies., Devil (s. Anm. 17), 115; I. Huber, Ansichten eines Zivilisierten über die unzivilisierte Welt: Das Sāsāniden-Bild des Georgios Pisides und sein historischer Wert für den spätantiken Iran, Klio 90 (2008), 162-192, hier 165.

25 Nissen (s. Anm. 11); Whitby (s. Anm. 10), 218 („George is first a man of God, and only second an imperial panegyrist“); dies., Devil (s. Anm. 17), 116; Huber (s. Anm. 24), 166f. Ob und in welcher Weise 
Georgios, der wahrscheinlich dem pisidischen Antiocheia entstammte, ${ }^{26}$ befand sich - möglicherweise mit Unterbrechungen ${ }^{27}$ - im Umfeld des Herakleios (er nahm wahrscheinlich an dessen Perserfeldzug 622 teil), ${ }^{28}$ pflegte aber auch ein enges Nahverhältnis zum Patriarchen Sergios, in dessen Auftrag er häufig dichtete und in dessen Klerikerstab er tätig war: Als Diakon diente er an der Hagia Sophia in Konstantinopel, wo er verschiedene Ämter bekleidete (skeuophylax, referendarius, chartophylax). In die Hauptstadt war er spätestens um 610/11 gelangt; damals jedenfalls entstand sein frühestes bekanntes Gedicht, ein kurzer Lobgesang auf Herakleios und seinen Putsch gegen Phokas (In Heraclium ex Africa redeuntem), ${ }^{29}$ mit dem der Dichter sich offenbar um Patronage von höchster Stelle bemühte. ${ }^{30}$ Die enge Verbindung theologischer und politischer Inhalte in Georgios' Werken manifestiert sich dann bereits anschaulich in seinem neuerdings auf das Jahr 613 datierten ${ }^{31}$ Ostergedicht In Christi Resurrectionem, das Herakleios' Sohn auf Kämpfe gegen Awaren und Perser vorbereitet, aber auch in den aktuellen Diskussionen um die politische Funktion des umfangreichen, insgesamt beinahe 2000 Verse umfassenden Hexaemeron. ${ }^{32}$ Ein Gedicht Contra Severum (629?) ${ }^{33}$ sollte die kaiserliche Religionspolitik unterstützen, während das Bellum Avaricum im Auftrag des Sergios die Rettung des belagerten Konstantinopel durch die Gottesmutter im Jahr 626 überhöht. In denselben Kontext gehört der Panegyricus In Bonum Patricium, ein verzweifelter Hilferuf an den Kaiser, die von Awaren und Persern eingeschlossene Hauptstadt, in der Georgios sich damals selbst befand, nicht im Stich zu lassen (626). Herakleios' Erfolge wiederum werden (wohl 623) in der Expeditio Persica (über den Feldzug 622) und insbesondere der Heraclias (entstanden nach dem 10. Mai 628) gepriesen - letzteres ein unvollendeter (ursprünglich wohl drei Gesänge umfassender) Abriss der größten Leistungen des Herakleios, den Georgios nach dem Triumph über die Perser verfasste - derselbe Ereigniszusammenhang bildet auch den Hintergrund der Dichtung In Restitutionem

man die angebliche Selbstcharakterisierung, die Georg. Pisid. Sev.695-699 gibt, ernst nehmen darf, ist noch völlig ungeklärt.

26 Whitby (s. Anm. 10), 201, mit Anm. 18.

27 Howard-Johnston (s. Anm. 17), 31-34, vermutet neuerdings, dass Georgios nach der Abfassung der Expeditio Persica (623) temporär (bis 629) die Gunst des Kaisers verloren habe (aufgrund einer unglücklichen Formulierung, die man als Anspielung auf Herakleios' Inzestehe mit dessen Nichte Martina habe interpretieren können).

28 Diese Vermutung basiert auf Georg. Pisid. Exped. Pers. 2,122-126; 3,131-136.

29 Zur Datierung: Frendo (s. Anm. 17), 167-171, mit ausführlicher Widerlegung des mittlerweile allgemein abgelehnten Datierungsvorschlags von Pertusi (s. Anm. 23), 18f. (619/20); Whitby (s. Anm. 10), 200; dies., Presentation (s. Anm. 17), 159; zuletzt Howard-Johnston (s. Anm. 17), 17.

30 Howard-Johnston (s. Anm. 17), 17.

31 So Howard-Johnston (s. Anm. 17), $18 \mathrm{f}$.

32 Dazu s. bes. Olster, Date (s. Anm. 17), der die politischen Implikationen zum Argument für einen neuen, späteren Datierungsvorschlag (638) macht, sowie die Replik von Whitby, Devil (s. Anm. 17). Das Hexaemeron dürfte über einen langen Zeitraum hin entstanden sein; seine Vollendung wird um 630 angesetzt.

33 So die Datierung durch Howard-Johnston (s. Anm. 17), 23. 
S. Crucis über die Rückführung der von den Persern 614 aus Jerusalem verschleppten Kreuzreliquien im Jahr 630. Reflexionen über das irdische Leben stellen schließlich die späten Dichtungen De vanitate vitae und De vita humana dar. Als letztes Erzeugnis gilt eine Prosavita des persischen christlichen Märtyrers Anastasios († 628) aus dem Jahr 632; ${ }^{34}$ kurz danach dürfte Georgios Pisides verstorben sein, jedenfalls deutet nichts darauf hin, dass er den Beginn der arabischen Expansion noch erlebt hätte. ${ }^{35}$ Seine insgesamt 115 überlieferten Epigramme entstanden wahrscheinlich als Parerga über die Jahre hin. Weitere Dichtungen lassen sich erschließen, sind aber nicht mehr erhalten $^{36}$ - so etwa eine Darstellung der frühen Regierungsjahre des Herakleios, ein Werk über die römischen Gegenoffensiven im Perserkrieg 624-626 und 627/28 oder auch die immerhin noch in einigen substanziellen Fragmenten greifbare dritte Akroasis der Heraclias. ${ }^{37}$

Als historische Quellen sind Georgios’ Dichtungen zwar von unschätzbarem Wert (insofern sie vielfach singuläre Informationen bieten), jedoch ausgesprochen schwer $\mathrm{zu}$ handhaben. ${ }^{38}$ Denn auch in den vermeintlich historisch-epischen Darstellungen wie der Expeditio Persica oder dem Bellum Avaricum gibt der Dichter keine zusammenhängenden Beschreibungen, sondern verfährt vielmehr impressionistisch, greift Einzelepisoden heraus, an denen bestimmte Leistungen und Eigenschaften der Protagonisten (zumeist des Kaisers) vorgeführt werden, und erschwert damit die Rekonstruktion von Ereigniszusammenhängen. Seine Dichtungen sind daher eher als Zeugnisse für die Repräsentation und Darstellung des Kaisers von Bedeutung, für die kaiserliche imago und für Erwartungen, mit denen der Herrscher konfrontiert wurde, aber auch für Hoffnungen und ,Stimmungen', die - etwa nach dem Sieg über die Sāsāniden 628 - offenbar gezielt gefördert wurden. Dabei bedient sich Georgios, den wir uns hochgebildet und mit der antiken Literatur wohlvertraut vorstellen müssen, eines relativ begrenzten Pools an literarischen Techniken und Motiven, die in der Forschung der letzten beiden Jahrzehnte aufgearbeitet worden sind. ${ }^{39} \mathrm{Zu}$ ihnen zählt die Synkrisis, d.h. die vergleichende Gegenüberstellung eines Protagonisten mit anderen Gestalten. ${ }^{40}$ Georgios wählt dabei eher selten Personen, die man im modernen

34 Edition und französische Übersetzung: B. Flusin, Saint Anastase le Perse et l'histoire de la Palestine au début du VII ${ }^{\mathrm{e}}$ siècle, Tome I: Les textes, Paris 1992, 202-259.

35 Vgl. Howard-Johnston (s. Anm. 17), $26 \mathrm{f}$.

36 Vgl. Pertusi (s. Anm. 23), 17-31; Howard-Johnston (s. Anm. 17), 25.

37 Die Frage, ob sich über die bei Theophanes und in der Suda identifizierbaren Georgios-Fragmente eine dritte Akroasis der Heraclias erschließen lässt, ist umstritten; einen Überblick über die Diskussionslage bietet Whitby, Presentation (s. Anm. 17), 168-172.

$38 \mathrm{Zu}$ den Möglichkeiten und Grenzen einer historischen Auswertung des Georgios Pisides s. HowardJohnston (s. Anm. 17), $30 \mathrm{f}$.

39 Vgl. dazu bes. Whitby, Presentation (s. Anm. 17), 157 f.; Howard-Johnston (s. Anm. 17), 21.

40 Nissen (s. Anm. 11), 301 ff.; Whitby (s. Anm. 10), 205. Eine kurze Definition der Synkrisis mit Blick auf die spätantike Literatur bietet C. Rapp, Comparison, Paradigm and the Case of Moses in Panegyric and Hagiography, in: Ma. Whitby (Hg.), The Propaganda of Power. The Role of Panegyric in Late Antiquity, Leiden/Boston/Köln 1998, 277-298, hier 279. 
Sinne als historisch bezeichnen würde: Homer, Demosthenes, Plutarch, Scipio, Alexander, Timotheos von Athen, Xerxes oder auch Konstantin I. ${ }^{41}$ Er schöpft vielmehr hauptsächlich aus einem üppigen Reservoir biblischer und mythischer Helden, wobei er kategorial kaum zwischen ihnen differenziert und keine Probleme damit hat, Personen aus dem Alten Testament Gestalten aus dem klassischen Mythos an die Seite zu stellen. ${ }^{42}$ Unter letzteren spielt die Figur des Herakles, wie bereits angedeutet, eine zentrale Rolle. ${ }^{43}$

\section{2.}

Mary Whitby glaubte die zentralen Funktionen der Herakles-Bezüge bei Georgios Pisides erfasst zu haben, als sie 1994 konstatierte: „It presents Heraclius as saviour and redeemer, justifying the violent overthrow of Phocas as the elimination of a bestial and corrupt force, and the wars against Persia as a crusade against an evil power with an alien religion. The Heracles image is ideal for the purpose because it stresses the violent, brutish and sub-human qualities of the opposition, and because Heracles is the great civilizing hero of classical mythology. “44

Begibt man sich daran, die einschlägigen Textbelege zu sichten, lässt sich diese Bewertung zunächst einmal bestätigen: So geht etwa aus einer insgesamt recht kryptischen Passage der Expeditio Persica zumindest so viel hervor, dass Herakles (= Herakleios) die Hydra besiegte (= Sieg über Chosroes), ohne sie jedoch bereits vernichtet zu haben - der Kontext (Herakleios’ Perserfeldzug 622) erscheint religiös aufgeladen, denn Georgios spricht von einem „vielköpfigen Irrtum“ der Hydra ( $\dot{\eta}$

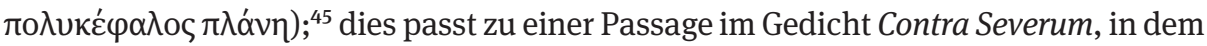
der Pisidier die zahlreichen Nacken (= Gruppierungen) der acephali (d.h. der Miaphysiten) beklagt, sie mit den Köpfen der Hydra vergleicht und seine Hoffnungen in

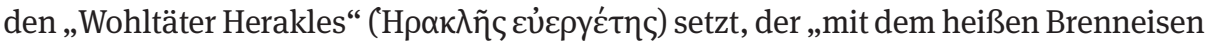

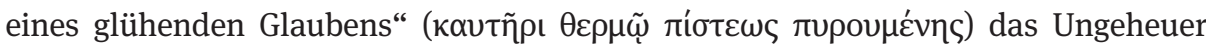

41 Vgl. Whitby (s. Anm. 10), 205, mit den Belegen.

42 Im Einzelnen s. dazu Whitby (s. Anm. 10), 206 ff., mit Diskussion der wichtigsten Exempla.

43 In diesem Sinne bereits Whitby (s. Anm. 10), 206: „The image recurs in almost all of George’s political poems, but it is used with remarkable versatility and variation.“

44 Whitby (s. Anm. 10), 208.

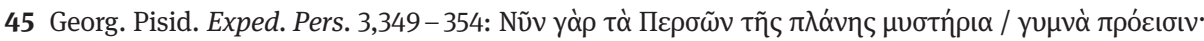

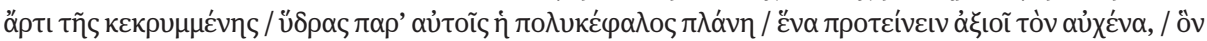

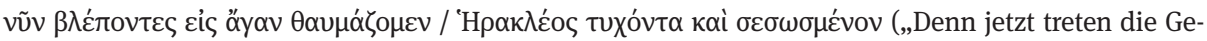
heimnisse des Irrtums [Irrglaubens?] der Perser enthüllt hervor; seit kurzer Zeit erachtet es der vielköpfige Irrtum der Hydra, die verborgen war, bei ihnen für recht, einen einzigen Nacken hervorzustrecken; und da wir diesen nun sehen, wundern wir uns außerordentlich, dass er mit Herakles zusammentreffen und sich dabei retten konnte“). Dazu s. den Kommentar von Pertusi (s. Anm. 23), $160 \mathrm{f}$. In den anschließenden Versen (355-359) äußert der Dichter die Hoffnung, dass es Herakleios in

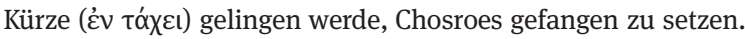


vernichte. ${ }^{46}$ Einmal mehr wird der Kampf gegen die Hydra dann im Bellum Avaricum apostrophiert, wobei das Ringen gegen ihre stets nachwachsenden Häupter hier wohl für die Beseitigung der durch das Missregiment des Phokas entstandenen Schäden und

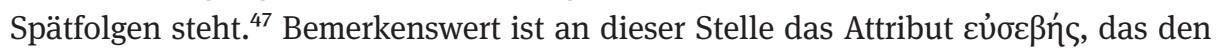
mythischen Helden mit einer zentralen Kaisertugend ausstattet, so dass in der For-

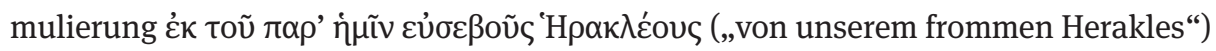
die Distanz zwischen Mythos und aktuellem Geschehen gänzlich aufgehoben erscheint: Herakles und Herakleios verschmelzen gleichsam zu einer überzeitlichen Entität. Dieselbe literarische Technik der Verkürzung wendet der Dichter an, wenn er in

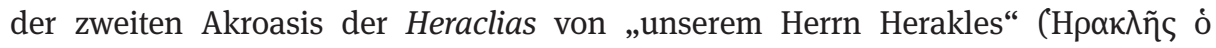

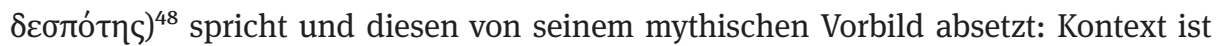

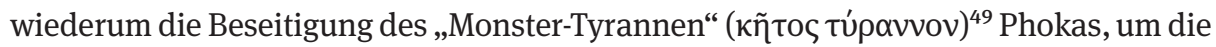
herum Georgios ein komplexes Netz aus Anspielungen webt, das Elemente des Perseus- und des Herakles-Mythos miteinander verbindet, ${ }^{50}$ um schließlich gegenüber den mythischen Exempla die universale Dimension der Aktionen des Kaisers zu betonen. ${ }^{51}$

Auf den ersten Blick bewegt sich Georgios mit Passagen wie den beschriebenen ganz im Rahmen traditioneller Mythenrezeption in der spätantiken Panegyrik. ${ }^{52}$ Whitbys Schlussfolgerung, dass Herakles vor allem als „saviour and redeemer“ präsentiert werde und dabei von seinem Kapital als großer Zivilisationsbringer lebe, ist insofern grundsätzlich zuzustimmen. Allerdings hat Whitby ebenfalls darauf hingewiesen, dass Georgios in der Art, wie er mit mythischen Exempla umgeht, eine erstaunlich große, jeweils kontextabhängige Variationsbreite erkennen lässt. ${ }^{53}$ Man denke dabei nur an die verschiedenen Konnotationen, mit denen er die Hydra zu verbinden vermag. Die Variatio kann dabei immerhin so weit gehen, dass etwa in dem Ostergedicht In Christi Resurrectionem gar der Sohn des Kaisers, Herakleios Konstantinos, auf Herakles' Kampf gegen die Hydra verwiesen wird (und zwar z.T. mit Versen, die nahezu identisch später in der Heraclias auf den Kaiser als Herakles bezogen werden). ${ }^{54}$ Generalisierungen werden damit schwierig; was sich für eine bestimmte Textpassage festhalten lässt, muss keineswegs auch für andere Stellen im Werk des Pisidiers gelten. Die von ihm entworfenen mythischen und biblischen Referenzen erweisen sich gewissermaßen als instabil, ja als fluide. Insofern lohnt es sich,

46 Georg. Pisid. Sev. 65-71.

47 Georg. Pisid. Bell. Avar. 49-57, mit Pertusi (s. Anm. 23), 211; Tartaglia (s. Anm. 17), 160, Anm. 9.

48 Georg. Pisid. Her. 2,21.

49 Georg. Pisid. Her. 2,22.

50 Georg. Pisid. Her. 2,12-23. Dazu s. H. Hunger, On the Imitation (MIMH $\mathrm{I} \Sigma$ ) of Antiquity in Byzantine Literature, DOP 23/24 (1969/70), 15-38, hier 24; Whitby (s. Anm. 10), 209.

51 Georg. Pisid. Her. 2,19-23.

52 So hatte insbesondere Nissen (s. Anm. 11), $301 \mathrm{ff}$., die Werke des Pisidiers beurteilt.

53 Whitby (s. Anm. 10), 208; dies., Devil (s. Anm. 17), 122.

54 Georg. Pisid. In Chr. Res. 106-111; Her. 1,78-79. Whitby, Devil (s. Anm. 17), 123; Howard-Johnston (s. Anm. 17), 19. 
genauer hinzusehen. Ich möchte im Folgenden einige Versgruppen zur Diskussion stellen, die über das Herakles-Exempel zwar ebenfalls Herakleios als Retter und Erlöser erscheinen lassen, dies jedoch in einer recht unkonventionellen Weise vermitteln - und die zugleich Implikationen beinhalten, die bisher noch nicht hinreichend beachtet worden sind.

Der mythische Held ist bereits in Georgios' frühestem Gedicht In Heraclium ex Africa redeuntem (610/11) präsent - ohne freilich namentlich genannt $\mathrm{zu}$ werden. Georgios Pisides stimmt ein Hohelied auf die geistig-spirituellen Fähigkeiten des Kaisers an und konfrontiert ihn dabei mit jenen, „denen nicht ein allweiser Verstand eingeprägt ist und die kein göttliches Wesen ziert“, nämlich den bewaffneten Reitern und treffsicheren Jägern. ${ }^{55}$ Die Herakles-Assoziation wird durch die Formulierung

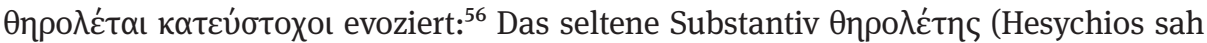

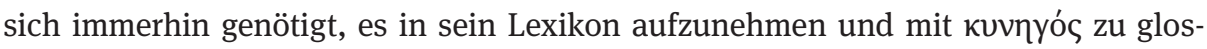
sieren) dient etwa auch in einem Epigramm der Anthologia Graeca zur Umschreibung

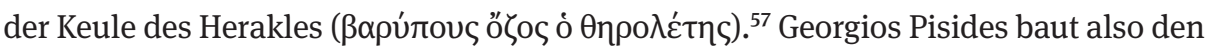
ungestümen „Monsterschlachter“ Herakles zum Gegenpol des besonnenen Kaisers auf (der ja auch gerade ein ,Monster‘, nämlich Phokas, geschlachtet hat, vgl.v. 39-62) und lässt ihn im Vergleich zu diesem zunächst einmal defizitär erscheinen; dies fällt jedoch nicht negativ auf den mythischen Helden zurück, denn niemand - nicht einmal ein Herakles - vermag eben die göttlichen Qualitäten eines Herakleios zu erreichen, wie der Dichter gleich eingangs konstatiert: „Ein Wort vermag dich nicht zu beschreiben, da ja das Wort Gottes selbst festgelegt hat, dass du über diese fließenden Worte gesetzt wurdest." 58

Das Motiv des Jagens wird auch im Folgenden weiterverfolgt - und damit die Herakles-Assoziation aufrecht erhalten: Der Kaiser setzt jeglicher Zornaufwallung wie wilden Tieren nach, jedoch aus einer Haltung der Milde heraus; ${ }^{59}$ er möchte die

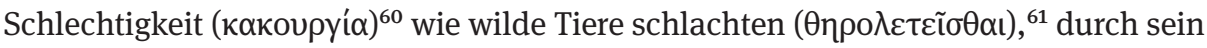

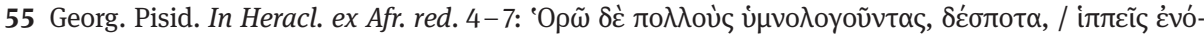

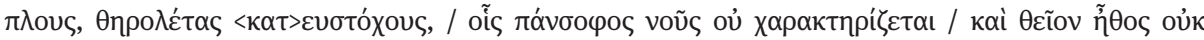

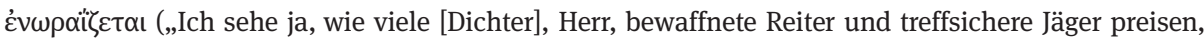

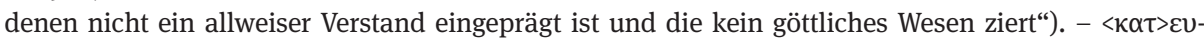
бтóxouৎ ist Konjektur Pertusis, vgl. dort den App. z. St.

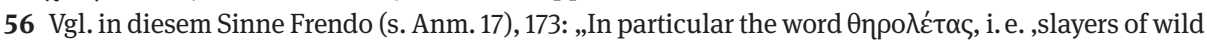
beasts or monsters' [...] suggests such heroes as Perseus and especially Heracles“; vgl. auch Whitby (s. Anm. 10), 211; dies., Presentation (s. Anm. 17), 160.

57 Anth. Graec. 16,104,4.

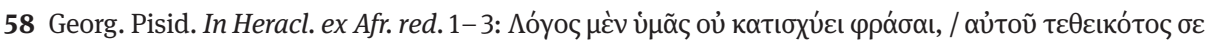

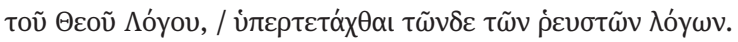

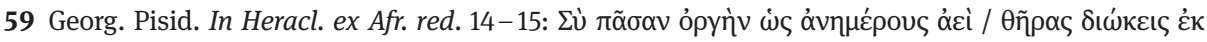

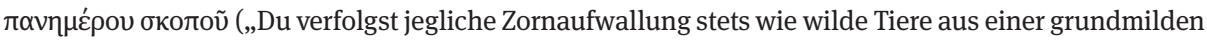
Haltung heraus").

60 Gemeint sein könnte Häresie im allgemeinen Sinne, vgl. Frendo (s. Anm. 17), 173 f.

61 Georg. Pisid. In Heracl. ex Afr. red. 16. 
Wesen werden Bestien gezähmt - konkret: die Perser und Barbaren (= Awaren) ${ }^{62}$ Die Grundaussage ist klar: Herakleios ist der bessere Herakles, und wie dieser spannt er seinen Bogen, aber aus dem Impetus der Philanthropia - also einer zentralen Kaisertugend - heraus. ${ }^{63}$

Ein längerer Abschnitt des Gedichts behandelt die Beseitigung des „Tyrannen“ Phokas durch Herakleios (v. 39-62). Insbesondere in diesen Versen wird die implizite Herakles-Herakleios-Analogie bemüht: Nicht nur, dass Phokas (mit apokalyptischem Subtext) als „herrschendes Tier“ bezeichnet und somit als Zielobjekt der Monsterjagd des Herakles-Herakleios gekennzeichnet wird; ${ }^{64}$ der Dichter rühmt den Kaiser überdies für seine bewusst getroffene Entscheidung, die Mühsal auf sich zu nehmen und den „Tyrannen“ zu beseitigen: „Du hättest die Gelegenheit gehabt, den Mühen ( $\pi$ óv $\omega v$ ) fernzubleiben, da du ja nicht schuld an unserem Unheil bist. “65 Das entsprechende Signalwort lautet róvor; es erscheint im Gedicht zweimal an prominenter Stelle (Versende) ${ }^{66}$ und verweist einmal mehr auf Herakles, dessen róvor in der Antike bekanntlich sprichwörtlich waren. ${ }^{67}$ Die Tatsache aber, dass die Entscheidung, den mühevollen Weg anzutreten, in derart deutlicher Weise als ein bewusster Akt hervorgehoben wird, deutet darüber hinaus auf die berühmte Erzählung von Herakles am Scheideweg als Referenzpunkt hin: ${ }^{68}$ Wie der mythische Held sah sich auch Herakleios vor die Wahl gestellt zwischen einem angenehmen, leichten Leben einerseits und einem Dasein voller Mühen - dieses aber achtbar und tugendhaft - andererseits. Und wie das mythische Vorbild wählte der Kaiser letzteres. ${ }^{69}$

Bereits in seinem ersten für uns greifbaren Gedicht arbeitet Georgios also mit dem Herakles-Exempel. Der mythische Held erscheint als implizite Vergleichsfolie für den Kaiser und dient dabei zu dessen Erhöhung. Herakles wird in seinen bekannten Rollen als Jäger wilder Monster sowie als vorbildlicher Held, der sich bewusst für ein tugendhaftes Leben voller Mühsal entscheidet, evoziert. In der erstgenannten Rolle besteht seine Funktion darin, die Vollkommenheit des Kaisers zu illustrieren: Dieser übertrifft sogar den archetypischen Heros schlechthin, weil er neben dessen ohnehin

62 Georg. Pisid. In Heracl. ex Afr. red.19-21. Die Verse enthalten eine subtile Anspielung auf Orpheus, der aber - wie Herakles - ebenfalls nicht namentlich genannt wird, vgl. Frendo (s. Anm. 17 ), 174.

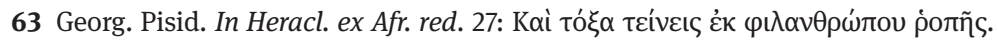

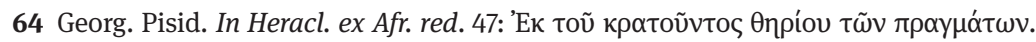

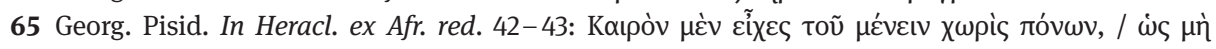

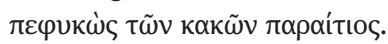

66 Georg. Pisid. In Heracl. ex Afr. red. 42; 48.

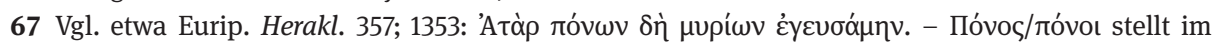
Schrifttum des Georgios eine bevorzugte Vokabel dar, für die sich - gerade im Bezug auf Herakleios zahlreiche Belege finden lassen. M.E. lässt sich daraus aber nicht folgern, dass der Terminus als Signalbegriff (u.a. mit Verweis auf Herakles) letztlich aussagelos sei; vielmehr könnte der Dichter auch die Intention verfolgt haben, das Herakles-Thema untergründig über weite Strecken hin präsent $\mathrm{zu}$ halten.

68 Prodikos D-K 84 B 2 = Xen. Mem. 2,1,21-34.

69 Georg. Pisid. In Heracl. ex Afr. red. 44-55. 
herausragenden Eigenschaften auch noch kluge Berechnung, Milde und Menschenfreundlichkeit mit einbringt. Der Rekurs auf die Geschichte von Herakles am Scheideweg hingegen konfrontiert uns einmal mehr mit überlegtem Handeln und verweist die Rezipienten darauf, dass Herakleios mit der Beseitigung des Phokas gezielt den Pfad der Tugend eingeschlagen hat. Was von Zeitgenossen auch als Verbrechen interpretiert werden konnte - nämlich ein Kaisermord -, gerät somit zur Urtat eines tugendhaften Helden als Folge seiner bewussten Entscheidung für das Gute.

Konsistent sind diese indirekten Rekurse auf die Herakles-Figur allerdings nicht, da der Held in unterschiedlicher Weise funktionalisiert wird: einmal als Kontrastfolie, ein anderes Mal als konkreter Anknüpfungspunkt mit Vorbildcharakter. Ohnehin ist anzunehmen, dass Georgios Pisides in dieser frühen Phase der Herrschaft des Herakleios - die um 610/11 auch noch keineswegs gesichert oder gar fest etabliert war ${ }^{70}$ zunächst einmal lediglich von der Namensassoziation Herakles-Herakleios ausgehen und dem Kaiser, dessen Patronage er ja ersstrebte, gewissermaßen Vorschläge bzw. Angebote machen konnte. Von einem bereits minutiös ausgearbeiteten ,Repräsentationsprogramm', das der Dichter in irgendeiner Weise aufgegriffen und reflektiert hätte, wird man für diesen frühen Zeitpunkt jedenfalls noch nicht ausgehen können.

Dies dürfte im Jahr 626, als der Pisidier aus dem belagerten Konstantinopel heraus einen eindringlichen Appell an den Kaiser verfasste, möglichst rasch vom Perserfeldzug in seine bedrohte Hauptstadt zurückzukehren, anders ausgesehen haben. Das zweigliedrige Gedicht In Bonum Patricium preist im ersten Teil (v. 1-48) die enge Verbindung zwischen Herakleios und seinem in Konstantinopel zurückgebliebenen Bevollmächtigten Bonos; ${ }^{71}$ der deutlich längere zweite Abschnitt (v. 49-170) enthält in immer neuen Variationen den pathetischen Rückkehr-Aufruf an Herakleios. Gleich im ersten Vers des Panegyricus wird das Herakles-Thema angesprochen, indem ein Vergleich zwischen der mythischen Vorzeit und dem aktuellen Augenblick gezogen

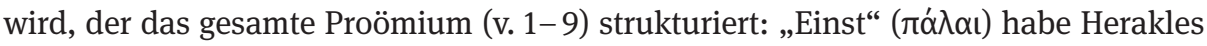
auf Befehl des Eurystheus eher geringe Aufgaben verrichten müssen; denn welchen Nutzen hätten die Tötung eines Ebers oder die Erdrosselung eines Löwen der Ge-

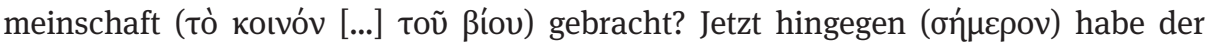

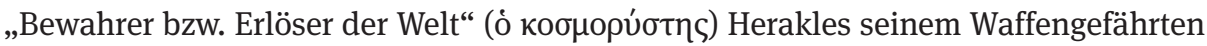
( $\tau \tilde{\omega}$ ouvó $\pi \lambda \omega)$ - dem im ersten Gedichtteil direkt angesprochenen Bonos - eine Welt in

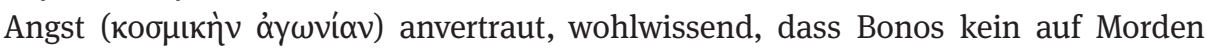

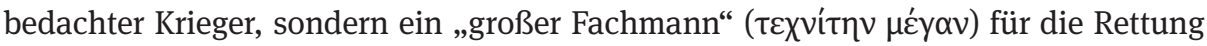

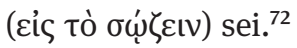

70 Vgl. W. E. Kaegi, New Evidence on the Early Reign of Heraclius, BZ 66 (1973), 308-330; ders., Heraclius. Emperor of Byzantium, Cambridge 2003, $58 \mathrm{ff}$;; D. M. Olster, The Politics of Usurpation in the Seventh Century: Rhetoric and Revolution in Byzantium, Amsterdam 1993, 18-21.

71 PLRE IIIA 242-244 (Bonus 5).

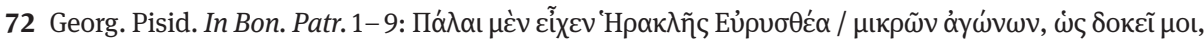

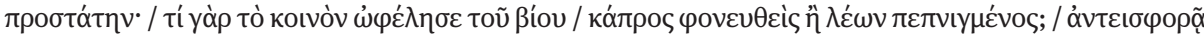

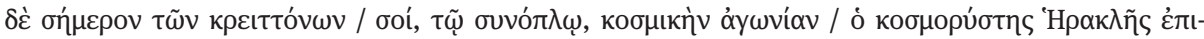


Zwei Punkte sind an diesem Proömium auffällig: Zum einen wird nun explizit die Gleichsetzung Herakles-Herakleios vollzogen; letzterer wird sogar ausdrücklich Herakles genannt (v. 7). Zum anderen erscheinen die róvoı (der Terminus wird im weiteren Verlauf des Gedichtes noch mehrfach fallen $)^{73}$ des mythischen Helden geradezu lächerlich im Vergleich mit jenen des Kaisers und seines Beauftragten - und dies nicht nur wegen der kosmischen Dimensionen, in denen Herakleios zu agieren hat (vgl. die

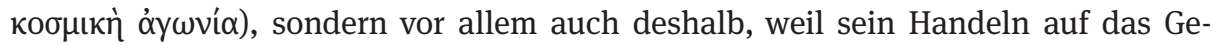
meinwesen bezogen ist und dabei nicht auf den Krieg als solchen, sondern auf die

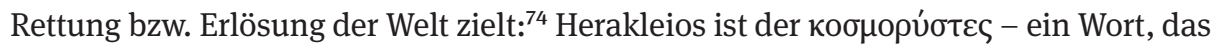
wohl von Georgios Pisides geprägt wurde (jedenfalls konnte ich keinen älteren literarischen Beleg ausmachen $)^{75}$ und dessen Semantik das im Neuen Testament ver-

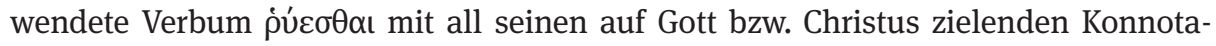
tionen evoziert. ${ }^{76}$ Angesichts dieser subtilen Überhöhung des Kaisers (durch die gezielte Herabsetzung des mythischen Herakles, der in der Antike selbstverständlich als Inbegriff des Zivilisationsbringers galt) ${ }^{77}$ lässt der Umstand aufhorchen, dass die auf das Proömium folgenden, die innige Verbindung zwischen Herakleios und Bonos preisenden Verse (v. 10 -48) Elemente zeitgenössischer theologischer Diskurse auf-

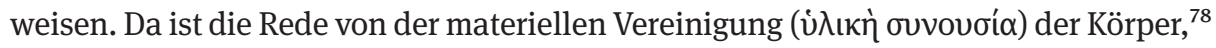
vom Fleisch, ${ }^{79}$ von der Henosis ${ }^{80}$ und vom Pneuma. ${ }^{81}$ Sachlich zielt der Dichter auf die Aussage, dass sich aufgrund des engen Nahverhältnisses zwischen dem Kaiser und seinem Vertrauten ( $\varepsilon \omega \omega !)^{82}$ letztlich eine einzige Seele in beiden Körpern befinde

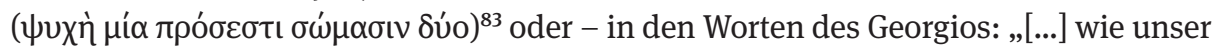

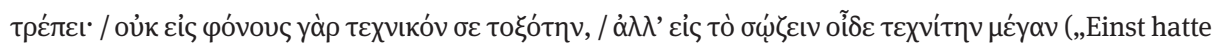
Herakles in Eurystheus einen Auftraggeber für geringe Aufgaben, wie mir scheint; denn was nutzte der menschlichen Gemeinschaft ein getöteter Eber oder ein erwürgter Löwe? Heute jedoch vertraut im Austausch für Größeres der Weltenerlöser Herakles Dir, seinem Waffengefährten, eine in Angst befindliche Welt an; denn er weiß, dass Du kein bogenbewehrter Krieger bist, der sich aufs Morden versteht, sondern ein großer Fachmann für die Rettung“).

73 Vgl. Georg. Pisid. In Bon. Patr. 14; 19; 57; 90; 91; 100; 101; 114; 115.

74 Vgl. Whitby (s. Anm. 10), $206 \mathrm{f}$.

75 Vgl. auch Olster, Roman Defeat (s. Anm. 17), 61; Tartaglia (s. Anm. 17), 199, Anm. 19.

76 Vgl. bes. 1 Thess 1,10; daneben etwa auch Mt 6,13; 27,43; Lk 1,74; Röm 7,24; 11,26; 15,31; 2 Kor 1,10; Kol 1,13; 2 Thess 3,2; 2 Tim 3,11; 4,17-18; 2 Petr 2,7; 2,9. Zur Beleglage s. W. Bauer, Griechisch-deutsches Wörterbuch zu den Schriften des Neuen Testaments und der frühchristlichen Literatur. 6., völlig neu bearbeitete Auflage hg. von K. Aland/B. Aland, Berlin/New York 1988, $1476 \mathrm{f}$.

77 Vgl. J. Trilling, Myth and Metaphor at the Byzantine Court. A Literary Approach to the David Plates, Byz 78 (1978), 249-263, hier 260.

78 Georg. Pisid. In Bon. Patr. 21; vgl. 26; 37.

79 Georg. Pisid. In Bon. Patr. 22.

80 Georg. Pisid. In Bon. Patr. 23.

81 Georg. Pisid. In Bon. Patr. 24.

82 Georg. Pisid. In Bon. Patr. 36.

83 Georg. Pisid. In Bon. Patr. 27. 
Herr [sc. der Kaiser] in dir [sc. Bonos] ist, auch wenn er weit entfernt zu sein scheint“ (

Mit dieser Feststellung gerät der gesamte zweite Teil des Gedichts letztlich zur Makulatur; denn der Appell, der Kaiser möge doch zurückkehren, ist überhaupt nicht erforderlich, da dieser ja in der Gestalt des Bonos bereits anwesend ist. Doch trotz seiner Präsenz, die zuvor mittels eines christologischen Vokabulars dargelegt wurde, ist er nicht sichtbar. Der Herr steht seinen Untertanen bei, aber man wünschte ihn sich unmittelbar fassbar. Geht es da noch um Herakleios? Geht es um Christus? Im Fol-

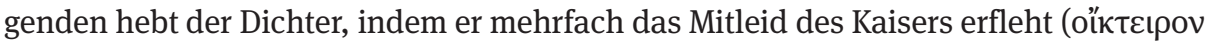
$\dot{\eta} \mu \tilde{\alpha} \varsigma),{ }^{85}$ auf die vergangenen Sünden der Konstantinopolitaner $a b^{86}$ und verweist darauf, dass Herakleios stets für alle die Mühen auf sich nehme: ${ }^{87}$ „Denn immer hast du dich für uns den Mühen unterworfen, da du für alle unsere Schwächen ertragen hast.“88 Ziel seines Wirkens ist nicht weniger als die „Rettung/Erlösung der Welt“

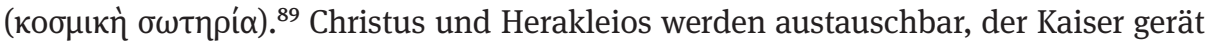
zum „Retter bzw. Erlöser der Welt“ - zum kosmorhýstes.

Diese Vorstellung - ganz unabhängig von der Frage, in welchem Maße sie vom Kaiser selbst forciert oder an ihn herangetragen wurde oder sich im kommunikativen Wechselspiel entwickelte - konnte im 7. Jahrhundert noch nicht vollkommen unverblümt artikuliert werden. Zwar sind die Andeutungen und Allusionen durchaus eindeutig, aber den letzten Schritt ist der Dichter dennoch nicht gegangen. ${ }^{90}$ Der kaiserliche, christusgleiche Weltenerlöser konnte nur in leichter Brechung als solcher erscheinen (auch wenn der Pisidier seine erhoffte Rückkehr offen als $\pi \alpha \rho o v \sigma i ́ \alpha$ bezeichnen konnte). ${ }^{91}$ Aus diesem Grunde dürfte Georgios zu Beginn des Panegyricus das

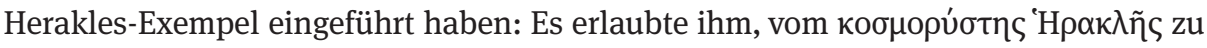
sprechen und den Namen des tatsächlich Gemeinten dabei vornehm zu verklausulieren, obwohl jeder Zuhörer wissen musste, um wen es ging. Der Bezug auf den Helden aus grauer Vorzeit garantierte das letzte noch verbliebene Moment an Distanz - jedenfalls vordergründig; auf einer subtileren Ebene hingegen hob er sie wieder auf und fundierte sogar die Herakleios-Christus-Analogie. Darauf ist noch zurückzukommen.

In der allgemeinen Jubelstimmung nach dem Eintreffen der Nachricht vom Tod des Perserkönigs Chosroes II. entstand in Konstantinopel seit 628 die Heraclias. Der Dichter verleiht zu Beginn des Epos dem Hochgefühl der Byzantiner beredten Aus-

84 Georg. Pisid. In Bon. Patr. 47-48.

85 Georg. Pisid. In Bon. Patr. 56; 60. Vgl. Röm 9,15.

86 Georg. Pisid. In Bon. Patr. 60 - 75.

87 Georg. Pisid. In Bon. Patr. 84-110.

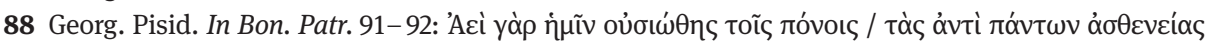
$\beta \alpha \sigma \tau \alpha ́ \alpha \alpha \varsigma$.

89 Georg. Pisid. In Bon. Patr. 123.

90 Vgl. in diesem Sinne auch Whitby (s. Anm. 10), 214, zu den Kaiser-Christus-Parallelen bei Georgios: „George stops short of explicit analogy between Heraclius and Christ, but only just.“

91 Georg. Pisid. In Bon. Patr. 121; vgl. Exped. Pers. 3,314. 
druck, indem er den Tod des „Feuerverehrers“ ( sich selbst für einen Gott gehalten habe, ${ }^{93}$ in Hybris verfallen sei (Georgios greift hier auf die Exempla Belsazars und des Xerxes zurück) ${ }^{94}$ und mit unbeschreiblicher Grausamkeit gewütet habe. ${ }^{95}$ Jetzt aber könnten der gesamte Kosmos, alle Elemente, aufatmen: „Doch Himmel, Erde, Feuer, Wasser, Luft und Wolken, ja der ganze Kosmos der überirdischen und unterirdischen Dinge applaudiert mit uns angesichts der Ratschlüsse Gottes, da einer gefallen ist und alle gerettet wurden."96

Mit dieser Einleitung verleiht der Dichter dem Folgenden einen kosmisch-religiösen Rahmen, ${ }^{97}$ innerhalb dessen auch der Vergleich zwischen Herakles und dem Kaiser zu deuten ist, der sich unmittelbar an den Triumphgesang ob des Todes des

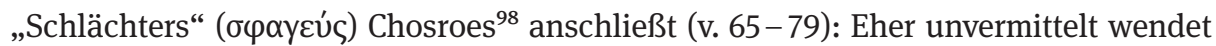
sich der Dichter nun direkt an Homer und attackiert ihn, weil er Herakles ohne Bedacht

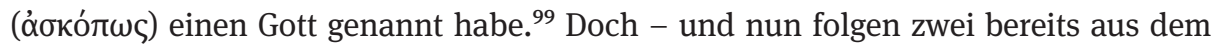
Panegyricus auf Bonos bekannte Verse - welchen Nutzen hätten die Tötung eines

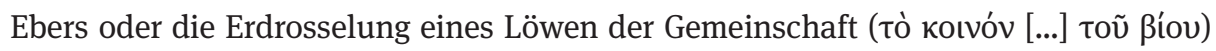
gebracht? ${ }^{100}$ Implizit folgt aus dieser Frage, dass nur ein Herakles, der auch dieser speziellen Anforderung - d.h. Nutzen für die Gemeinschaft zu erbringen - gerecht wird, mit gutem Grund als Gott erscheinen mag. Und tatsächlich fordert Georgios nun,

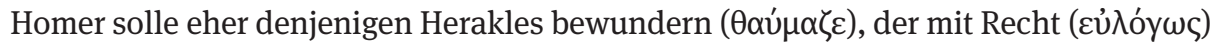
kosmorhýstes heiße. ${ }^{101}$ Einmal mehr wird hier der Kaiser mit der terminologischen Neuprägung, die ihn in die Nähe zu Christus rückt, beschrieben, wobei die Kombi-

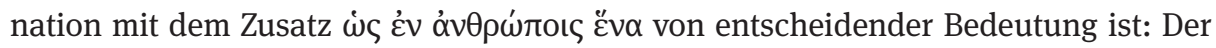

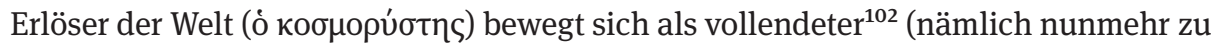
Recht als Gott zu bezeichnender) Herakles unter den Menschen. Die Christus-Analogie ist hier offenkundig, und einmal mehr wird sie durch den Herakles-Bezug leicht verklausuliert, zugleich aber auch indirekt bestätigt. Denn Herakles lebte ebenfalls als

92 Georg. Pisid. Her. 1,14; vgl. 1,181. Huber (s. Anm. 24), 127.

93 Georg. Pisid. Her. 1,22-23, mit Pertusi (s. Anm. 23), 263; Huber (s. Anm. 24), 179.

94 Georg. Pisid. Her. 1,31-35 (Belsazar; vgl. Tartaglia [s. Anm. 17], 197, Anm. 11); 1,27- 30 (Xerxes; vgl. Exped. Pers. 2,303-305).

95 Georg. Pisid. Her. 1,36-48; Huber (s. Anm. 24), $178 \mathrm{f}$.

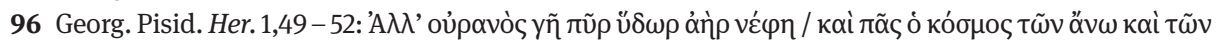

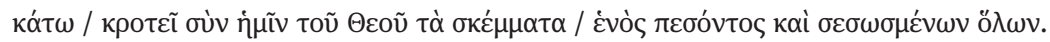

97 Vgl. Hunger (s. Anm. 50), 24, der von einem „Kreuzzug“ („crusade“) spricht.

98 Georg. Pisid. Her. 1,64.

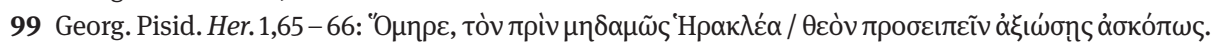
Der Vorwurf beruht wohl auf einem Missverständnis von Odysseus’ Begegnung mit Herakles in der Nekyia der Odyssee (Od. 11,601-603).

100 Georg. Pisid. Her. 1,67-68 = In Bon. Patr. 3-4.

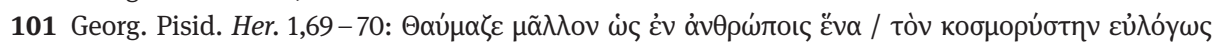

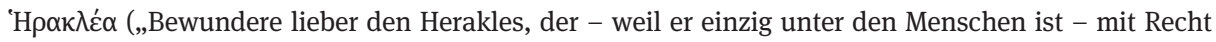
Erlöser der Welt [genannt wird]“).

102 Vgl. pointiert Trilling (s. Anm. 77), 260: „Heraclius is not only compared to Herakles, he is Herakles. In fact he is even more Herakles than Herakles himself.“ 
Sohn Gottes und einer Sterblichen unter den Menschen. In den folgenden Versen werden einige seiner mythischen Großtaten auf Herakleios hin gedeutet (ein in der Panegyrik geläufiges Verfahren). ${ }^{103}$ Georgios nennt exemplarisch Herakles’ Abstieg in den Hades (= Herakleios' Vordringen ins Perserreich), ${ }^{104}$ die Bezwingung des Kerberos (= Chosroes), ${ }^{105}$ die Wiedererweckung der Alkestis (= Wiederherstellung der christlichen Oikoumene), ${ }^{106}$ die Tötung des Drachen (der die Äpfel der Hesperiden bewachte = Chosroes), ${ }^{107}$ die Vernichtung der Hydra (= Chosroes), ${ }^{108}$ die Reinigung des Augiasstalles (= Wiederherstellung einer ehrbaren Lebensführung? ${ }^{109}$ und die Erdrosselung des Nemeischen Löwen (= Chosroes). ${ }^{110}$ „Und nun“, so der Dichter weiter, ,ist Herakles in seinem Werk fortgeschritten, da er sich die Goldenen Äpfel nahm - alle Städte [sc. die zuvor von den Persern besetzt gewesen waren]““.111

Bemerkenswert sind indes die nun folgenden Verse: „Es verzog sich die Quelle des finsteren Abends, Licht verbreitete sich und die Finsternis verschwand“ - wir befinden uns plötzlich mitten im Schöpfungsbericht (vgl. Gen 1,4-5) - und weiter: „Und ein

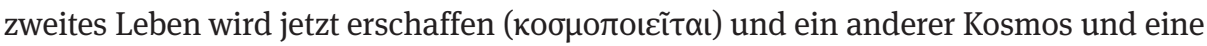
erneuerte Schöpfung (кті́бıৎ).“112 Die von Georgios geknüpfte Assoziationskette ist

103 Georg. Pisid. Her. 1,71-79, mit Nissen (s. Anm. 11), 302f. Die Kriterien für die von Georgios getroffene Auswahl erschließen sich nicht unmittelbar, und bei einigen Beispielen lässt sich die Frage, in welcher Weise sie auf Herakleios hin zu deuten sind, nicht mit letzter Sicherheit beantworten. Whitby (s. Anm. 10), 207, Anm. 55, warnt daher zu Recht davor, „to press the historical allusions of specific labours“ - auch deshalb, weil Georgios sie z.T. in anderen Kontexten auch anders verwendet. Ich richte mich im Folgenden nach den Deutungen, die Tartaglia (s. Anm. 17), 199, Anm. 20 - 21, vorschlägt.

104 Georg. Pisid. Her. 1,71; vgl. Apollod. Bibl. 2,122-126.

105 Georg. Pisid. Her. 1,72; vgl. Apollod. Bibl. 2,125-126.

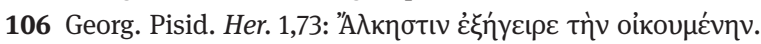

107 Georg. Pisid. Her. 1,74; vgl. Apollod. Bibl. 2,113; 121. Ob der Dichter tatsächlich alle von Herakles besiegten Ungeheuer einzig auf Chosroes gedeutet wissen wollte, erscheint mir zumindest unsicher (auch wenn der Kontext der ersten Akroasis der Heraclias dies nahelegt). M.E. wäre mitunter durchaus auch an den gestürzten „Tyrannen“ Phokas zu denken. So bezeichnet der Dichter etwa nicht nur

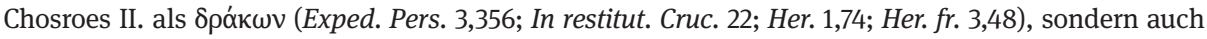
seinen Vorgänger (Bell. Avar. 50). Huber (s. Anm. 24), 179f., versteht indes sämtliche bei Georg. Pisid. Her. 1,71-79 genannten Monster als Anspielungen auf Chosroes.

108 Georg. Pisid. Her. 1,75; vgl. Apollod. Bibl. 2,77-80.

109 Georg. Pisid. Her. 1,76; vgl. Apollod. Bibl. 2,88-91.

110 Georg. Pisid. Her. 1,77; vgl. Apollod. Bibl. 2,74-75. Georgios bezeichnet Chosroes hier als ,Zerstörer

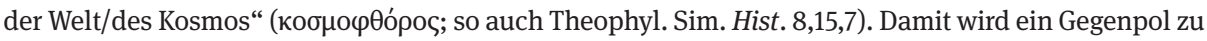

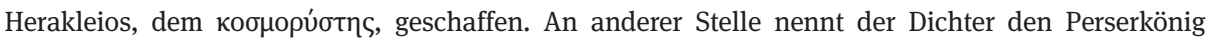

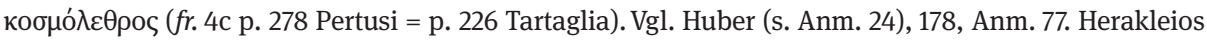

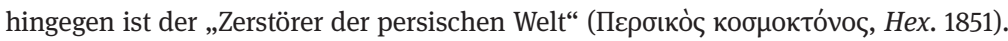

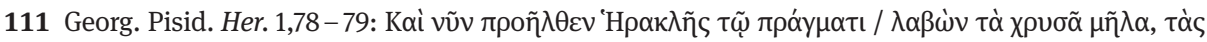

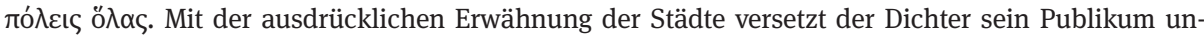
vermittelt wieder in die aktuelle Situation und macht dabei deutlich, dass er die zuvor genannten Taten des Herakles tatsächlich auf zeitgenössische Vorgänge bezogen wissen wollte.

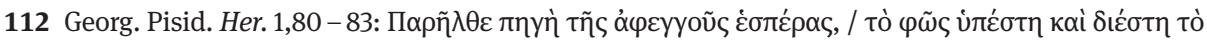

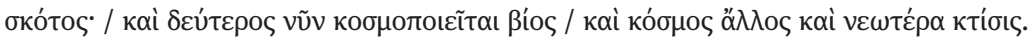


damit bei der Sintflut angelangt. Folgerichtig erscheint Herakleios in den folgenden

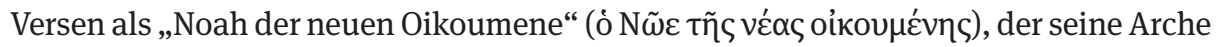
in seinem Herzen gefunden habe, ${ }^{113}$ wohingegen Chosroes mit der Sintflut identifiziert wird. ${ }^{114}$ Mit deutlichen Bezügen auf den biblischen Sintflutbericht betont der Pisidier einmal mehr die Verdorbenheit und Sündhaftigkeit der Menschen - bis Herakleios-

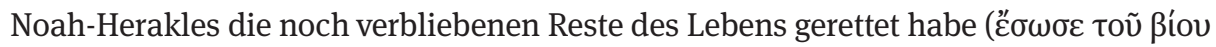
$\tau \dot{\alpha} \lambda \varepsilon^{\prime}(\psi \alpha v \alpha) .{ }^{115}$

Georgios Pisides arbeitet - dies sollte deutlich geworden sein - vielfach mit recht freien Assoziationsketten, die nicht immer modernen Ansprüchen an präzise Textstrukturierung genügen. Es geht dem Dichter indes auch weniger darum, unbedingt über längere Partien hin die Kohärenz seiner Vergleiche und Analogien aufrecht zu erhalten, sondern sein Vorgehen ist eher als impressionistisch anzusehen: Er entwirft Bilder, die wiederum spezifische Assoziationen evozieren, mit denen dann weiter gearbeitet wird. So dürfte der abrupte Übergang von Herakles zu Noah und der Sintflut ${ }^{116}$ wahrscheinlich nur beim modernen Rezipienten Verwunderung auslösen, der gewohnt ist, zwischen biblischen und mythischen Figuren zu differenzieren; ${ }^{117}$ in Byzanz machte die Kombination mythischer und biblischer Beispiele dagegen sogar Schule. ${ }^{118}$ Innerhalb des Gesamtzusammenhangs der ersten Akroasis der Heraclias steht jedoch zunächst einmal der Triumph über die Niederringung des Sāsānidenreiches im Vordergrund, der ganz auf den Kaiser zentriert und entsprechend ausgestaltet wird. Vor einem in höchstem Maße religiös aufgeladenen Hintergrund wird Herakleios dabei in zweifacher Weise präsentiert: Als Retter bzw. Erlöser der Welt (durch Auslöschung des gefährlichsten auswärtigen Gegners) erscheint er in der Rolle eines neuen, sich unter den Menschen bewegenden Gottes, des vollendeten Herakles. Als Neugestalter der Oikoumene hingegen, als Initiator einer erneuerten Schöpfung, die aufgrund menschlicher Sündhaftigkeit erforderlich wurde, zeigt er sich als „Noah der neuen Oikoumene“. Das dabei entstehende Amalgam aus mythischen und christlichen Bezügen zielt jedoch insgesamt vor allem auf eines: Die HerakleiosChristus-Analogie. Denn nicht nur der kosmorhýstes Herakles barg entsprechende Implikationen; auch Noah wurde typologisch auf Christus hin gedeutet. ${ }^{119}$ Sowohl der Herakles-Rekurs als auch das Noah-Exempel bewirken damit denselben Effekt.

Die Herakles-Bezüge im Panegyricus auf Bonos und im ersten Gesang der Heraclias spielen also mit der Herakleios-Christus-Parallele, indem sie einerseits auf diese hindeuten, sie andererseits aber auch leicht verschleiern. Dass es sich dabei

113 Georg. Pisid. Her. 1,84-85.

114 Georg. Pisid. Her. 1,88.

115 Georg. Pisid. Her. 1,89-92; vgl. Gen 6,12; 6,17; 7,1-24; 8,11.

116 Das Herakles-Motiv klingt allerdings später (Georg. Pisid. Her. 1,94) noch einmal an, wenn der Dichter erneut die róvos erwähnt.

117 Trilling (s. Anm. 77), 260; Whitby (s. Anm. 10), 212.

118 Hunger (s. Anm. 50), $23 \mathrm{f}$.

119 Vgl. etwa Origen. Hom. in Gen. 2,3; Joh. Chrysost. De Lazaro Conc. VI PG 48,1037. 
keineswegs um singuläre Ausnahmefälle im Werk des Pisidiers handelt, wird deutlich, wenn man die bereits angesprochene Passage aus der Expeditio Persica genauer betrachtet und vor allem ihren Kontext mit berücksichtigt: Dem Hinweis auf den Sieg des Herakles-Herakleios gegen die irrgläubige Hydra Chosroes geht eine Passage voraus, in welcher der Kaiser sich aufgrund der awarischen Bedrohung gezwungen sieht, von der Perserfront nach Konstantinopel zurückzukehren, und dementsprechend umsichtige Maßnahmen trifft. ${ }^{120}$ Das Herakles-Motiv wird dabei bereits subtil angedeutet, indem Georgios wiederholt auf die freiwillige Übernahme schwerer róvoı durch Herakleios hinweist ${ }^{121}$ und die Feinde der Byzantiner als wilde Tiere bezeichnet, die es zu jagen gelte. ${ }^{122}$ Gleichzeitig aber mischt sich noch eine weitere Assoziation in die Darstellung: Christus. So wird des Kaisers bevorstehende Rückkehr in „die Polis“ als $\pi \alpha \rho o v \sigma i ́ \alpha$ bezeichnet ${ }^{123}$ und - was noch weitaus deutlicher ist - der Herrscher schlüpft nun in die Rolle des guten Hirten, und zwar nicht irgendeines guten Hirten, sondern des Erzhirten

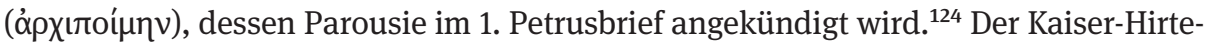
Vergleich zieht sich über mehrere Verse hin (v. 3,322-335) und wird dabei geschickt mit den bereits genannten Herakles-Assoziationen verwoben. ${ }^{125}$ Herakleios ist Herakles dies wird in v. 3,354 offen ausgesprochen. Aber er ist auch Christus: Wenige Zeilen,

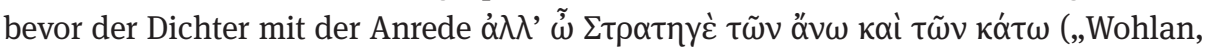
Gebieter über Himmel und Erde!“") sein großes Schlussgebet für den Kaiser und seine Nachkommen beginnt, ${ }^{126}$ wendet er sich noch einmal direkt an Herakleios. Die Anrede

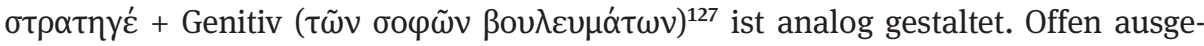
sprochen wird die Parallele zwar nicht (ja, sie wird später sogar wieder leicht relativiert); ${ }^{128}$ aber jeder zeitgenössische Zuhörer wird sie vernommen haben: Georgios präsentiert am Ende der Expeditio Persica einen Herakles-Herakleios-Christus.

120 Georg. Pisid. Exped. Pers. 3,305-340.

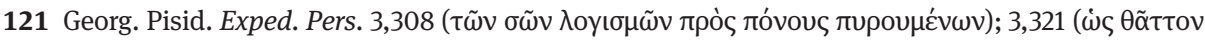

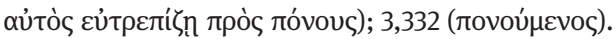

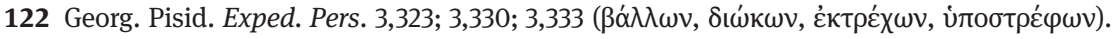

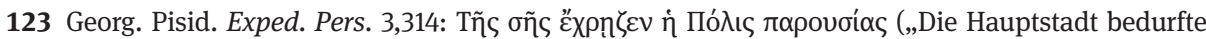
deiner Parousie“).

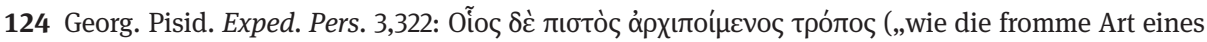

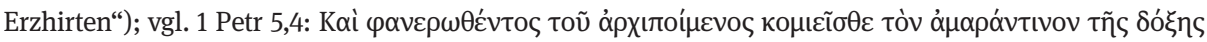

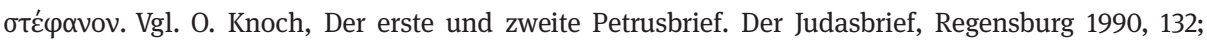
R. Feldmeier, Theologischer Handkommentar zum Neuen Testament, Bd. XV.1, Leipzig 2005, $157 \mathrm{f}$. 125 Hirten und Herde: Georg. Pisid. Exped. Pers. 3,324; 3,327; 3,328; 3,335.

126 Georg. Pisid. Exped. Pers. 3,385-461.

127 Georg. Pisid. Exped. Pers. 3,374.

128 Die Parallelisierung wird später behutsam zurückgenommen, wenn der Dichter den Kaiser als

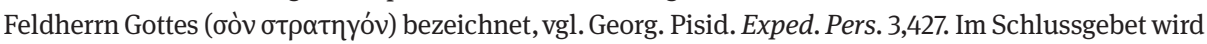
Herakleios zudem in die Rolle eines Neuen Moses gerückt (diese Parallele wurde seit Konstantin verschiedentlich gezogen, vgl. Euseb. VC 1,12; 1,20,2; 1,38,2; 2,12,1; dazu Rapp [s. Anm. 40], 292-295; B. Isele, Moses oder Pharao? Die ersten christlichen Kaiser und das Argument der Bibel, in: A. Pečar/K. Trampedach [Hgg.], Die Bibel als politisches Argument. Voraussetzungen und Folgen biblizistischer Herrschaftslegitimation in der Vormoderne, München 2007, 103-118; Georgios parallelisiert auch 
Wie gesehen, ist für die über das Herakles-Exempel vermittelte Christus-Assoziation der wohl von Georgios in die Literatur eingeführte Terminus des kosmorhýstes, des „Retters bzw. Erlösers der Welt“, von einiger Relevanz. Im Schrifttum des Pisidiers begegnet diese Neuprägung insgesamt viermal: Im Panegyricus auf Bonos und in der Heraclias - über beide Passagen habe ich bereits gehandelt - sowie im Gedicht Contra Severum und im Hexaemeron. ${ }^{129}$

Der Beleg im Hexaemeron findet sich kurz vor dem Ende des monumentalen Gedichts in einem Gebet an Gott, das Georgios pathetisch mit den Worten „Oh, Ar-

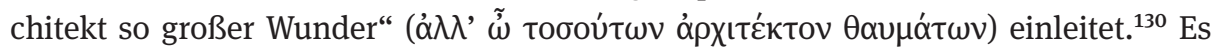
folgen eine Parallelisierung der Tore Konstantinopels mit den Pforten des Himmels ${ }^{131}$

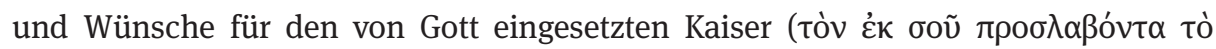

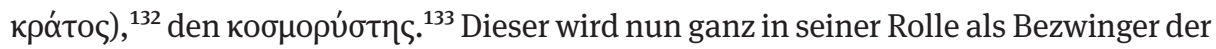
Perser präsentiert; ${ }^{134}$ er möge alle Orte unter der Sonne beherrschen, denn es sei

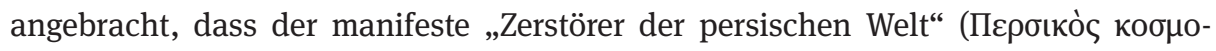

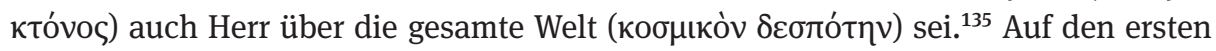

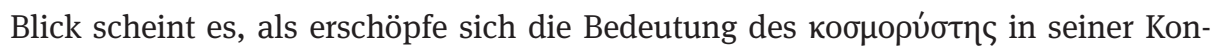
kretisierung als „Zerstörer der persischen Welt“ - ganz so, wie in der Heraclias der

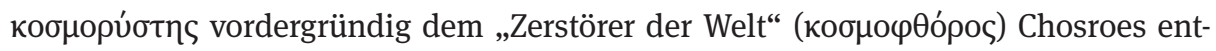
gegengestellt wird. ${ }^{136}$

Allerdings hatte der Autor den unmittelbar vorausgehenden Abschnitt ebenfalls mit den identischen Worten „Oh, Architekt so großer Wunder“ eingeleitet und darauf einen überschwänglichen Christus-Hymnus angestimmt. ${ }^{137}$ Für den Leser des Gedichts war also, als er im Anschluss daran ein zweites Mal dieselbe Anredeformel zur Kenntnis nehmen musste, der Christus-Bezug noch unmittelbar präsent - auch wenn es nunmehr um den Kaiser ging; doch konnte jetzt mit dem geschickt platzierten

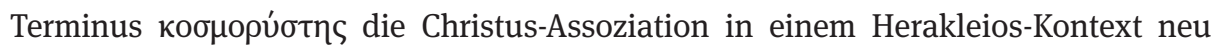
aktiviert werden. Auch im Hexaemeron dient das Wort also dazu, implizit das Herakleios-Christus-Muster zu evozieren.

Die einzigartige Vollkommenheit des Herakleios, aufgrund derer ihn Georgios wie gezeigt - mehrfach von seinem Vorbild Herakles abgehoben hatte, dominiert

\footnotetext{
Sergios mit Moses, vgl. Bell. Avar. 495-501; Hex. 1828), wobei Georgios sein enges Nahverhältnis zu Gott und Christus hervorhebt (Exped. Pers. 3,415-425). Zum Vergleich des spätantiken Kaisers mit Moses s. Rapp (s. Anm. 40), bes. 295f. (zu Herakleios).

129 Georg. Pisid. In Bon. Patr. 7; Her. 1,70; Sev. 452; Hex. 1800 (ed. Tartaglia).

130 Georg. Pisid. Hex. 1792ff. (ed. Tartaglia). Zu dieser Passage vgl. Olster, Date (s. Anm. 17), 168f.; Whitby, Devil (s. Anm. 17), 118-120.

131 Georg. Pisid. Hex. 1793-1798 (ed. Tartaglia).

132 Georg. Pisid. Hex. 1799 (ed. Tartaglia).

133 Georg. Pisid. Hex. 1800 (ed. Tartaglia).

134 Vgl. bes. Georg. Pisid. Hex. 1800-1801 (ed. Tartaglia).

135 Georg. Pisid. Hex. 1800-1806 (ed. Tartaglia).

136 Georg. Pisid. Her. 1,77. S.o. Anm. 110.

137 Georg. Pisid. Hex. 1720 - 1791 (ed. Tartaglia).
} 


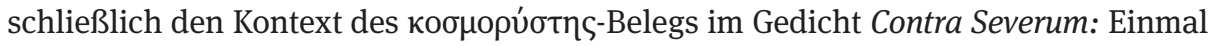
mehr wird der Kaiser als erfolgreicher Bezwinger der Perser präsentiert: „der kosmorhýstes, der Schlächter des Chosroes, unser Bollwerk, der Richter über Persien“

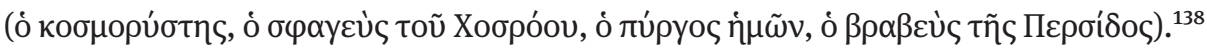
Aber es bleibt eben nicht bei diesen militärischen Qualitäten: Herakleios „besänftigt erstens mit Waffen und zweitens mit Worten alle wilden Tiere in ihrem Gemüt“ (тò

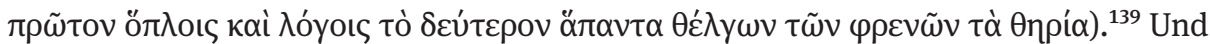
daher werde er, so Georgios weiter, auch die Severianer noch überzeugen. ${ }^{140}$

Die herausragenden Qualitäten des Kaisers manifestieren sich also in allen Bereichen: dem Krieg, dem vernunftgemäßen Handeln, der Religion. Es ist gerade diese Vollkommenheit, die den kosmorhýstes Herakleios vor allen anderen Wesen - auch vor einem mythischen Zivilisationsbringer wie Herakles - in besonderer Weise auszeichnet, und es ist diese Eigenschaft, die ihn in unmittelbare Nähe zu Christus rückt.

\section{3.}

Bezüge zwischen Herakleios und Christus, sei es in Form von Analogien, Parallelen oder auch nur leisen Andeutungen, lassen sich in der Dokumentation zu diesem Kaiser allenthalben nachweisen, so dass ich mich im Folgenden kurz fassen kann. Das Phänomen selbst ist in der Forschung mittlerweile bekannt, ${ }^{141}$ wenngleich noch nicht systematisch aufgearbeitet worden. Insbesondere das Schrifttum des Georgios Pisides dürfte bei einer genaueren Analyse ohne Zweifel noch weiteres einschlägiges Belegmaterial erbringen. Doch schon jetzt ist auch bei diesem noch wenig erforschten Autor manches offenkundig, ${ }^{142}$ so etwa die erwähnte Darstellung des Herakleios als ó $\rho{ }^{-}$ $\pi o i ́ \mu \eta v$ Christus ${ }^{143}$ oder auch die Beschreibung des kaiserlichen adventus in Jerusalem anlässlich der Rückführung der Kreuzreliquien, die eindeutig Christi Einzug in die heilige Stadt evoziert. ${ }^{144}$ In der Expeditio Persica weckt die sprachliche Ausgestaltung der Schilderung einer blutenden Fußverletzung des Kaisers Assoziationen an die Passion Christi, ${ }^{145}$ ebenso wie in der Heraclias die triumphale Rückkehr des Augustus

138 Georg. Pisid. Sev. 452-453.

139 Georg. Pisid. Sev. 454-455.

140 Georg. Pisid. Sev. $456-457$.

141 J. W. Drijvers, Heraclius and the Restitutio Crucis. Notes on Symbolism and Ideology, in: G. J. Reinink/B. H. Stolte (Hgg.), The Reign of Heraclius (610 - 641). Crisis and Confrontation, Leuven/Paris/ Dudley (Ma.) 2002, 175-190, bes. $184 \mathrm{ff}$.

142 Vgl. Whitby (s. Anm. 10), 214.

143 Vgl. Whitby (s. Anm. 10), $213 \mathrm{f}$.

144 Vgl. Georg. Pisid. In restitut. Cruc. 5-8; dazu Mt 21,8 - 9; Joh 12,13; Frendo (s. Anm. 17), 180, Anm. 78; Olster, Date (s. Anm. 17), 161; Whitby (s. Anm. 10), 215; dies., Presentation (s. Anm. 17), 161 („Heraclius' arrival in Jerusalem is analogous to that of Christ on Palm Sunday“).

145 Georg. Pisid. Exped. Pers. 2,239-247, mit Trilling (s. Anm. 77), 259f.; Whitby (s. Anm. 10), 205, Anm. 41; 215; dies., Presentation (s. Anm. 17), 163, mit Anm. 36. 
nach Konstantinopel mit impliziten Christus-Bezügen operiert. ${ }^{146}$ Ganz unverhohlen wird schließlich in einer Theophanes-Passage, die auf die verlorene dritte Akroasis der Heraclias zurückgeht, Herakleios mit Gott parallelisiert: „Nachdem der Kaiser aber in sechs Jahren Persien niedergerungen hatte, schloss er im siebten Jahr Frieden und kehrte unter großem Jubel nach Konstantinopel zurück, wobei er damit eine mystische Schau erfüllte. Denn nachdem Gott in sechs Tagen die gesamte Schöpfung vollbracht hatte, nannte er den siebten ,Ruhetag'. So auch der Kaiser: Nachdem er in sechs Jahren viele Mühen auf sich genommen hatte, wandte er sich im siebten Jahr in Frieden und Freude der Hauptstadt zu und ruhte.“147

Der Pisidier bewegte sich mit derartigen Evokationen allerdings keineswegs erratisch in einer ansonsten ganz anders geprägten Umgebung, sondern griff - im Gegenteil - offenbar verbreitete Tendenzen auf. ${ }^{148}$ In diesem Diskussionszusammenhang spielt auch die Übernahme des Basileus-Titels durch Herakleios nach dem Sieg über die Perser eine Rolle: Der Kaiser und sein Sohn Herakleios Konstantinos erscheinen in

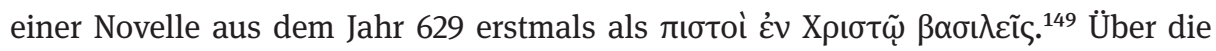
Gründe für diese Reform ist in der Forschung viel spekuliert worden. ${ }^{150}$ Zuletzt hat Otto Kresten dafür plädiert, die Motive des Kaisers als ein komplexes Amalgam aus DavidBezügen, Assoziationen im Zusammenhang der Rückführung des Kreuzes nach Jerusalem und messianisch-eschatologischen Elementen zu deuten: Demzufolge habe Herakleios den Restitutionsakt mit der Überführung der Bundeslade durch König David parallelisiert, um dem Alten Bund zwischen Gott und dem auserwählten Volk einen Neuen Bund (mit den Römern) an die Seite zu stellen; aus diesem Grund habe er den davidischen Königstitel basileús angenommen. ${ }^{151}$ Sollte sich diese Deutung als zutreffend erweisen, dann wären die inhärenten Christus-Parallelen offenkundig. ${ }^{152}$

146 Georg. Pisid. Her. 1,192-218, mit Whitby (s. Anm. 10), 215f.; dies., Devil (s. Anm. 17), $119 ; 121$.

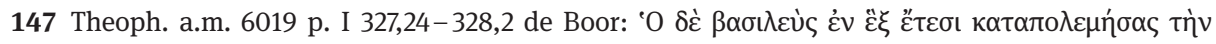

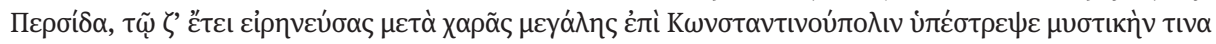

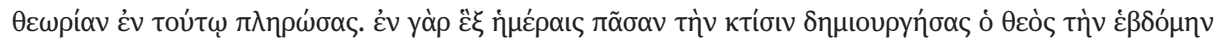

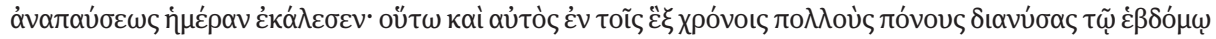

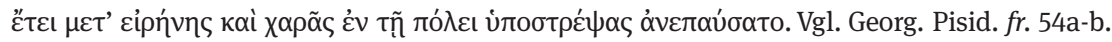

148 So auch Whitby (s. Anm. 10), $216 \mathrm{ff}$.

149 J. Konidaris, Die Novellen des Kaisers Herakleios, in: D. Simon (Hg.), Fontes Minores V, Frankfurt a. M. 1982, 33-106, hier 84; zur Datierung der Novelle ebd., $58 \mathrm{f}$.

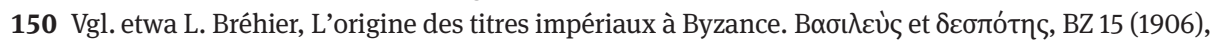
161-178, bes. 172-178; I. Shahîd, The Iranian Factor in Byzantium during the Reign of Heraclius, DOP 26 (1972), 293-320; E. K. Chrysos, The Title BA II $\Lambda$ EY $\Sigma$ in Early Byzantine International Relations, DOP 32 (1978), 29-75.

151 O. Kresten, Oktateuch-Probleme: Bemerkungen zu einer Neuerscheinung, BZ 84/85 (1991/92), 501511, hier 504, Anm. 13; ders., Herakleios und der Titel $\beta \alpha \sigma \iota \lambda \varepsilon u ́ c$, in: Varia VII (= Poikila Byzantina 18), Bonn 2000, 178-179; vgl. auch Shahîd (s. Anm. 150), 307 f.; S. Spain Alexander, Heraclius, Byzantine Imperial Ideology, and the David Plates, Spec 52 (1977), 217-237, bes. 232-234; M. Mundell Mango, Imperial Art in the Seventh Century, in: P. Magdalino (Hg.), New Constantines. The Rhythm of Imperial Renewal in Byzantium, 4th-13th Centuries, Aldershot 1994, 109-136, hier 128; Rapp (s. Anm. 40), 295; W. Brandes, Heraclius between Restoration and Reform. Some Remarks on Recent Research, in: G. J. 
Herakleios wurde indes ohnehin vor allem über die David-Typologie (vgl. 2 Sam 7,12-13) auf Christus bezogen. ${ }^{153}$ Die Gleichsetzung des Kaisers mit David ist u. a. in der Homilie auf die Belagerung Konstantinopels 626, die der Zeitzeuge Theodoros Synkellos verfasste, in hohem Maße präsent. ${ }^{154}$ Als herausragende Evidenz für die besondere Relevanz der David-Thematik gelten in der Forschung allerdings die vieldiskutierten Silberteller mit David-Motiven („David plates“), die „were manufactured on the order of Heraclius to promulgate the new ideology of his reign“. ${ }^{155}$ Möchte man Georgios Pisides glauben (der sich ansonsten auffallend zurückhaltend gegenüber der David-Typologie zeigt), ${ }^{156}$ so hat Herakleios das David-Exempel sogar selbst vor seinen Soldaten vorgebracht. ${ }^{157}$ Ganz unwahrscheinlich erscheint dies nicht angesichts der Tatsache, dass der Kaiser seinen 630 geborenen Sohn auf den Namen David taufen ließ und auch damit ein deutliches Signal setzte. ${ }^{158}$ Die David-Rekurse des Herakleios waren immerhin derart zahlreich und prominent, dass sie selbst im fernen Franken-

Reinink/B. H. Stolte (Hgg.), The Reign of Heraclius (610 - 641). Crisis and Confrontation, Leuven/Paris/ Dudley (Ma.) 2002, 17-40, bes. 19. Das Kreuz als neue Bundeslade: Georg. Pisid. In restitut. Cruc. 71-77, mit Tartaglia (s. Anm. 17), 244f., Anm. 16; s. auch Ludwig (s. Anm. 17), 97 ff. - Der Titel basileús ist allerdings - entgegen der immer wieder anzutreffenden Behauptung - nicht ganz neu unter Herakleios, sondern vereinzelt auch schon früher belegt, so etwa für Konstantin I. (OGIS 721 = SEG 37, 1650 [326 n. Chr.]), für Constantius II. (TAM III.1, 13 [= R. Heberdey (Ed.), Tituli Pisidiae linguis Graeca et Latina conscripti. Tituli Termessi et Agri Termessensis, Wien 1941]) und für Arkadios (ISmyrna II.1 [IK 24.1], 845) [Hinweise von Christoph Begass].

152 Vgl. Y. Stoyanov, Defenders and Enemies of the True Cross. The Sasanian Conquest of Jerusalem in 614 and Byzantine Ideology of Anti-Persian Warfare, Wien 2011, $66 \mathrm{f}$.

$153 \mathrm{Zu}$ den Rekursen des Herakleios auf David vgl. etwa Spain Alexander (s. Anm. 151), 229-234; Ludwig (s. Anm. 17), 93 ff.; dies., David - Christus - Basileus. Erwartungen an eine Herrschergestalt, in: W. Dietrich/H. Herkommer (Hgg.), König David - biblische Schlüsselfigur und europäische Leitgestalt, Stuttgart u.a. 2003, 367-382, bes. 370; 373-378; Mundell Mango (s. Anm. 151), 122-131; Drijvers (s. Anm. 141), 184f.; Kaegi, Emperor (s. Anm. 70), 114; 139. Der von R. Stichel, Scenes from the Life of King David in Dura Europos and in Byzantine Art, Jewish Art 23/24 (1997/98), 100 - 116, bes. 100 -103, und U.

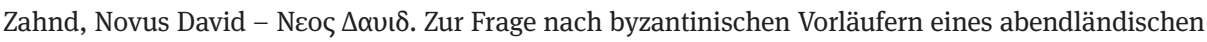
Topos, FMSt 42 (2008), 71-87, bes. 80 - 83, unternommene Versuch, die Relevanz des David-Exempels unter Herakleios infrage zu stellen, wirkt angesichts der überwältigen Evidenz wenig überzeugend. 154 Vgl. etwa Theod. Synk. 2-5 p. 298,19-299,39; 38 p. 313,32-37; 52 p. 320,20-21 (= F. Makk, Traduction et commentaire de l'homélie écrite probablement par Théodore le Syncelle sur le siège de Constantinople en 626. Appendice: Analecta Avarica de L. Sternbach, Szeged 1975); Ludwig (s. Anm. 17), 95-97.

155 Drijvers (s. Anm. 141), 185. Zu den ,David plates‘ s. etwa Spain Alexander (s. Anm. 151); Trilling (s. Anm. 77); Mundell Mango (s. Anm. 151), 122-131; Whitby (s. Anm. 10), 218f.; Ludwig (s. Anm. 153), 370; 373-378. Kritik an dem bis vor kurzem als selbstverständlich vorausgesetzten Zusammenhang dieser Objekte mit der kaiserlichen Selbstdarstellung äußern R. E. Leader, The David Plates Revisited: Transforming the Secular in Early Byzantium, Art Bulletin 82 (2000), 407-427; V. Tsamakda, König David als Typos des byzantinischen Kaisers, in: F. Daim/J. Drauschke (Hgg.), Byzanz - Das Römerreich im Mittelalter, Teil 1: Welt der Ideen, Welt der Dinge, Mainz 2010, 23-54, bes. 30 - 33.

156 Vgl. Whitby (s. Anm. 10), 218f., mit Anm. 107; Howard-Johnston (s. Anm. 17), 32, mit Anm. 47-48. 157 Georg. Pisid. Exped. Pers. 2,113-115.

158 PLRE IIIA 390 (David 8). 
reich beim sog. Fredegar reflektiert wurden. ${ }^{159}$ Für Herakleios bot sich David als Referenzpunkt aus mehreren Gründen an: Beide Herrscher gelangten auf eher ,unkonventionelle ' Weise auf den Thron; beide bekämpften Tyrannen und Ungläubige; beide pflegten ungehörige Beziehungen zu Frauen (David zu der verheirateten Bathseba, Herakleios zu seiner Nichte Martina); beide unterwarfen sich und ihre Herrschaft bedingungslos dem göttlichen Willen. Beide schließlich - dieser Aspekt dürfte nach 630 von besonderer Bedeutung gewesen sein - transferierten heilige Objekte an ihren Ort: David die Bundeslade, Herakleios die Kreuzreliquien. ${ }^{160}$

\section{4.}

Insbesondere über die vielfältigen David-Bezüge lassen sich somit die Christus-Assoziationen des Herakleios erfassen - und auf diese Weise konnten sie in der Repräsentation auch nachdrücklich vermittelt werden, ohne allzu großen Anstoß zu erregen. In einer ähnlichen Weise wird man die Verwendung des Herakles-Exempels $\mathrm{zu}$ beurteilen haben, denn für Zeitgenossen war auch in diesem Fall die ChristusParallele augenfällig. Ich kann mich auch in diesem Punkt kurz fassen, denn die auf Christus verweisende Herakles-Typologie ist jüngst von Henrike Maria Zilling luzide herausgearbeitet worden: ${ }^{161}$ „Beide sind Göttersöhne und werden von sterblichen Müttern geboren; beide vollbringen in den Augen der Nachwelt verehrungswürdige, außergewöhnliche Taten und Wunder, beide sterben einen grausamen Tod und beide fahren in den Himmel auf, in das Reich ihrer göttlichen Väter. “162 Die Abstammung des mythischen Helden vom höchsten Gott und einer sterblichen Frau fügte sich nahtlos in die spätantike Zweinaturenlehre. ${ }^{163}$ Insbesondere aber musste sein „Leiden als Mensch vor der Rückkehr zum göttlichen Vater“ (auf dem Berg Oita) für christliche Zeitgenossen entsprechende Assoziationen hervorrufen. ${ }^{164}$ Angesichts dieser manifesten Parallelen nimmt es nicht wunder, dass christliche Autoren Herakles, der bei Dion Chrysostomos immerhin als soter und Herrscher über das gesamte Menschengeschlecht erscheinen konnte ${ }^{165}$ und in der Bevölkerung überdies ungemein populär war, als Konkurrenz wahrnahmen. Scharfe Polemiken resultierten daraus, in denen vor allem seine burlesken Seiten, sein ungehemmtes Saufen, Fressen und seine sexuelle Zügellosigkeit (d.h. gerade jene Eigenschaften, die ihn so beliebt gemacht

159 Vgl. Fredeg. 4,64: Aeraclius imperatur arma sumens, telam priliae et phalange a suis postergum preparatum relinquens, singolare certamen, ut novos Davit, procedit ad bellum. S. H. Wander, The Cyprus Plates and the Chronicle of Fredegar, DOP 29 (1975), $345 \mathrm{f}$.

160 Drijvers (s. Anm. 141), 185; vgl. auch Ludwig (s. Anm. 17), 95.

161 Zum Folgenden s. H. M. Zilling, Jesus als Held. Odysseus und Herakles als Vorbilder christlicher Heldentypologie, Paderborn u.a. 2011, $119 \mathrm{ff}$.

162 Zilling (s. Anm. 161), 153.

163 Zilling (s. Anm. 161), $151 \mathrm{f}$.

164 Zilling (s. Anm. 161), 161.

165 Dion Chrys. 1,84. 
hatten), hervorgehoben und attackiert wurden. ${ }^{166}$ Freilich bin ich nicht davon überzeugt, dass Herakles „diesen Kampf schließlich verloren“ habe, wie Zilling unter Berufung auf eine Äußerung aus dem frühen 5. Jahrhundert, wonach Herakles in Rom nicht mehr als Gott verehrt werde, konstatiert. ${ }^{167}$ Etwa um dieselbe Zeit entstand immerhin der 6. Hymnos des Synesios, in dem Christus mit dem (namentlich nicht genannten) Herakles direkt parallelisiert wird: Wie Herakles reinigte Christus Land, Meer, Luft, die Tiefe der Erde und stieg in den Hades hinab. ${ }^{168}$ Überdies hat Zilling selbst herausgearbeitet, wie Prodikos' Erzählung von Herakles am Scheideweg in sublimierter Form in die christliche Zwei-Wege-Lehre eingehen konnte und den Heros damit weiterhin als Folie präsent hielt. ${ }^{169}$ Herakles wurde also in der Tat zwar nicht mehr als Gott verehrt, aber er barg dennoch ein Potential, das in den vielfältigen Analogien zu Geburt, Leben und Leiden Christi beruhte - und das jederzeit aktiviert werden konnte, um auf Christus zu verweisen. Als mythischer Held gehörte Herakles im 7. Jahrhundert zum angestaubten Bildungsschatz einer jahrhundertealten Tradition; man konnte dieses gelehrte Wissen abrufen und demonstrieren - wie das eingangs erwähnte anonyme Herakles-Gedicht zeigt. ${ }^{170}$ Aber ein christlicher Kaiser, der sich wie einst Commodus oder die Tetrarchen direkt auf Herakles als mythisches Vorbild und Gottheit berufen und diesen gar imitiert hätte, hätte sich im Kontext des Imperium Christianum doch wohl eher lächerlich gemacht. Wenn man das Exempel dennoch abrief, dann konnte die Sinngebung nur noch innerhalb der veränderten Rahmenbedingungen, d.h. vor einem christlichen Hintergrund erfolgen - und damit wurden unweigerlich die altbekannten Herakles-Christus-Parallelen aktiviert.

\section{5.}

Die abschließende Frage, warum Herakleios in einer bis dahin singulären Intensität mit Christus assoziiert wurde - weitaus nachdrücklicher als etwa Konstantin oder Justinian $^{171}$-, bedarf einer eigenständigen Untersuchung und kann an dieser Stelle

166 G. K. Galinsky, The Herakles Theme. The Adaptations of the Hero in Literature from Homer to the Twentieth Century, Oxford 1972, 4; 189; Zilling (s. Anm.161), 121;189ff.; 222f. Aus dieser Haltung konnten durchaus gewalttätige Auseinandersetzungen resultieren, wie aus Augustin. epist. 50 Goldbacher hervorgeht: Nach der Zerstörung von Heraklesbildnissen im afrikanischen Sufeta durch christliche Eiferer kam es zu Ausschreitungen, bei denen die Christen 60 Tote zu beklagen hatten, vgl. Zilling (s. Anm. 161), 197.

167 Zilling (s. Anm. 161), 151, unter Hinweis auf Augustin. serm. 24,6 (= C. Lambot [Ed.], Sancti Aurelii Augustini Sermones de Vetere Testamento [CCSL 41], Turnhout 1961, p. 332).

168 Synes. Hymn. 6,33-39, mit dem Kommentar von J. Gruber/H. Strohm, Synesios von Kyrene. Hymnen. Eingeleitet, übersetzt und kommentiert, Heidelberg 1991, 217; vgl. dazu auch Hunger (s. Anm. 50), 22f., der darauf hinweist, dass die Herakles-Christus-Parallele auch ikonographisch greifbar ist.

169 Vgl. Zilling (s. Anm. 161), $208 \mathrm{ff}$.

170 Vgl. Hunger (s. Anm. 8), II 110: „reiner Lern- und Merkstoff für die Schule im weitesten Sinne“. 171 Vgl. Rebenich (s. Anm. 16); Meier, Zeitalter (s. Anm. 16), 547 ff. 
daher nur noch perspektivisch angerissen werden. Von zentraler Bedeutung scheint mir in diesem Zusammenhang die in spätjustinianischer Zeit einsetzende sog. ,Liturgisierung‘ zu sein, d.h. die Durchdringung sämtlicher für uns fassbarer Lebensbereiche der oströmisch-byzantinischen Gesellschaft mit christlich-religiösen Inhalten und entsprechender Symbolik. ${ }^{172}$ In dem Maße, in dem die Gesellschaft des Imperium Romanum, ja dieses selbst, eine wachsende sakrale Aufladung erfuhr, musste auch der Kaiser, der als Beauftragter Gottes ja eine sichtbare Distanz zu seinen Untertanen zu wahren hatte, einen neuerlichen Sakralisierungsprozess durchlaufen. ${ }^{173}$ Hatte schon Justinian die Parallelen zwischen Kaiser und Christus bemüht, so war für die nachfolgenden Kaiser angesichts der sich wandelnden Rahmenbedingungen kaum noch ein Zurückgehen hinter die bereits bestehenden Marken möglich - eher das Gegenteil war der Fall.

Im speziellen Fall des Herakleios dürften noch weitere Faktoren hinzugekommen sein: Die blutige Beseitigung des Phokas in einem langwierigen Bürgerkrieg hatte ebenso wie dessen eigene Usurpation die etablierte Ordnung massiv infrage gestellt: ${ }^{174}$ Seit Konstantin das Imperium Christianum geschaffen hatte, hatte es keinen erfolgreichen Thronräuber im Osten mehr gegeben, wie der Kirchenhistoriker Euagrios noch gegen Ende des 6. Jahrhunderts mit besonderem Nachdruck festgehalten hatte. ${ }^{175}$ Nicht nur Phokas war also ein Zerstörer der seit nunmehr rund 300 Jahren geltenden Ordnung gewesen, sondern auch Herakleios. Umso mehr musste dieser sich darum bemühen, diesen Makel abzustreifen. Ich habe an anderer Stelle nachzuweisen versucht, dass der Kaiser sich wohl aus diesem Grunde immer wieder als Restitutor der alten Ordnung präsentierte, ja dass seine eigene Repräsentation offenbar Teil eines übergreifenden Ordnungsdiskurses war, der aufgrund der Kaisermorde 602 und 610 mit einiger Intensität geführt wurde. ${ }^{176}$ Die Ordnung war aber auch aus anderen Gründen bedroht: Im ersten Jahrzehnt der Herrschaft des Herakleios verlor das Oströmische Reich so viele Territorien wie nie zuvor; die römische Weltherrschaft schien an ihr Ende gelangt. ${ }^{177}$ Auch auf diese Herausforderungen musste der Kaiser reagieren, und dies konnte nicht nur auf militärischem Weg geschehen. Mit den persischen Erfolgen dürften sich - wir können dies punktuell fassen - eschatologische Naherwartungen verbreitet haben (so wie kurz darauf auch im Kontext der arabischen Ex-

172 Zur ,Liturgisierung، s. v.a. Av. Cameron, Images of Authority: Elites and Icons in Late Sixth-Century Byzantium, P\&P 84 (1979), 3-35, ND in: dies., Continuity and Change in Sixth-Century Byzantium, London 1981, XVIII.Vgl. auch M. Meier, Sind wir nicht alle heilig? Zum Konzept des „Heiligen“ (sacrum) in spätjustinianischer Zeit, Millennium 1 (2004), 133-164, mit weiterer Literatur (133, Anm. 1); Meier (s. Anm. 1).

173 Dazu im Einzelnen demnächst M. Meier, Liturgification and Hyper-Sacralization: The Declining Importance of Imperial Piety in Constantinople between the $6^{\text {th }}$ and $7^{\text {th }}$ Centuries A.D. (im Druck). 174 Dazu ausführlich M. Meier, Kaiser Phokas (602-610 n.Chr.) als Erinnerungsproblem, BZ 107 (2014), 139-174.

175 Euagr. $H E$ 3,41.

176 Meier (s. Anm. 174).

177 Überblick bei Kaegi, Emperor (s. Anm. 70), 58 ff. 
pansion), die unweigerlich auch den Kaiser in den Fokus rückten. ${ }^{178}$ Nicht zuletzt mit der Inszenierung der Rückführung der Kreuzreliquien nach Jerusalem im Jahr 630 dürfte dieser auch auf Anmutungen dieser Art reagiert haben. Innerhalb eines solchen Amalgams aus generellen Verdichtungen religiöser Ausdrucksformen im gesamten Imperium Romanum, aus persönlich-situativen Erfordernissen, aus den gewaltigen außen- (und innen-)politischen Herausforderungen und aus eschatologischen Naherwartungen dürfte die traditionelle Kaiser-Christus-Parallele in besonderem Maße an Relevanz gewonnen haben; wie bereits angedeutet, dürfte diese, komplexe Kommunikationsprozesse voraussetzende Entwicklung sicherlich kaum von Herakleios allein ausgegangen sein. Aber er wird versucht haben, ihre Richtung und Wirkungsweise wo irgend möglich zu beeinflussen. Georgios Pisides als Dichter in seinem Umfeld könnte ihm dabei behilflich gewesen sein, indem er gezielt das Herakles-Exempel aktualisierte und dem Kaiser damit eine Möglichkeit anwies, die Christus-Parallelen einerseits in unmissverständlicher Weise zu evozieren, sie dabei gleichzeitig aber auch so zu verschleiern, dass die letzten Grenzen des Sagbaren nicht überschritten wurden.

178 Einführend vgl. dazu W. Brandes, Die apokalyptische Literatur, in: F. Winkelmann (Hg.), Quellen zur Geschichte des frühen Byzanz (4.-9. Jahrhundert). Bestand und Probleme, Berlin 1990, 305-322. 